University of Rhode Island

DigitalCommons@URI

Open Access Dissertations

2014

\title{
TOWARDS STEINBERG'S CONJECTURE
}

Diana L. Smith

University of Rhode Island, dianasmith15@gmail.com

Follow this and additional works at: https://digitalcommons.uri.edu/oa_diss

\section{Recommended Citation}

Smith, Diana L., "TOWARDS STEINBERG'S CONJECTURE" (2014). Open Access Dissertations. Paper 212. https://digitalcommons.uri.edu/oa_diss/212

This Dissertation is brought to you for free and open access by DigitalCommons@URI. It has been accepted for inclusion in Open Access Dissertations by an authorized administrator of DigitalCommons@URI. For more information, please contact digitalcommons-group@uri.edu. 
TOWARDS STEINBERG'S CONJECTURE

BY

DIANA L. SMITH

A DISSERTATION SUBMITTED IN PARTIAL FULFILLMENT OF THE REQUIREMENTS FOR THE DEGREE OF

DOCTOR OF PHILOSOPHY

IN

MATHEMATICS

UNIVERSITY OF RHODE ISLAND

2014 
DOCTOR OF PHILOSOPHY DISSERTATION

$\mathrm{OF}$

DIANA L. SMITH

APPROVED:

Dissertation Committee:

Major Professor Nancy Eaton

Jessica Libertini

Joan Peckham

Nasser H. Zawia

DEAN OF THE GRADUATE SCHOOL

\section{UNIVERSITY OF RHODE ISLAND}

2014 


\begin{abstract}
Several conjectures concerning planar graph colorings are still unsolved to this day. One of the more famous ones is Steinberg's Conjecture (first stated in 1976), which we work towards in this dissertation. Steinberg's Conjecture states that all planar graphs without cycles of length 4 or 5 are 3 -colorable, that is, we can color the vertices of such a graph using three colors in a way that leaves no adjacent vertices colored the same.

We use defective colorings to move closer to the affirmation or nullification of this conjecture. A defective coloring is any non-proper coloring, that is, some adjacent vertices may end up colored the same color. We use $\left(d_{1}, d_{2}, d_{3}\right)$-colorings, which are 3-colorings where the maximum degree of the $i^{\text {th }}$ color class is at most $d_{i}$, for $i=1,2,3$. In this study, we prove that all planar graphs without cycles of length 4 or 5 are $(3,0,0)$-colorable.

We define a cycle having two triangular chords as 2-chorded and a face of size $k$ as a $k$-face. Let a $b_{8}$-face be an 8 -face incident to a vertex of degree three, which is itself also incident to faces of size 3 and 6 . Let $\mathcal{G}$ be the set of all planar graph without $C_{4}$ 's, $C_{5}$ 's, 2-chorded $C_{8}$ 's, or 2-chorded $C_{9}$ 's. In this paper, we prove that all graphs in $\mathcal{G}$ with at most eleven $b_{8}$-faces is $(1,0,0)$-colorable.

We end this dissertation by examining a paper claiming to prove that all planar graphs without 5- and 8-cycles and without adjacent triangles are 3-colorable. We show some counterexamples to a claim given in the paper.
\end{abstract}




\section{ACKNOWLEDGMENTS}

I would first and foremost like to acknowledge my adviser Dr. Nancy Eaton

for all her advice and support over the past several years. Her door has always been open to me through the nine years I have known her. I do not think I would have made it this far without her help. She has always been willing to lend an ear and to help boost my self confidence.

I would also like to thank my Defense Committee members: Dr. Nancy Eaton, Dr. Edmund Lamagna, Dr. Jessica Libertini, Dr. Joan Peckham, and Dr. Luboš Thoma. They all graciously agreed to take time out of their busy schedules to read and review my dissertation, and I thank them all sincerely for their words of advice.

I would like to thank the graduate students and faculty of the University of Rhode Island for making these last few years fly by. This second family of mine has always been very supportive and helpful. It will be very strange and surreal not to see them all on a daily basis anymore.

And last but certainly not least, I would like to thank my family for all their love and support throughout my life. 


\section{DEDICATION}

I would like to dedicate this work to my family: My mother Linda, my father Thomas, my sisters Christine and Carolyn, and my brothers Joseph and David. 


\section{TABLE OF CONTENTS}

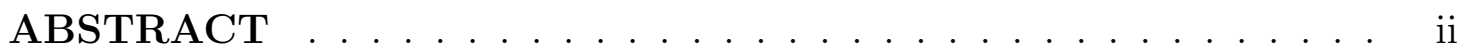

ACKNOWLEDGMENTS ................. . iii

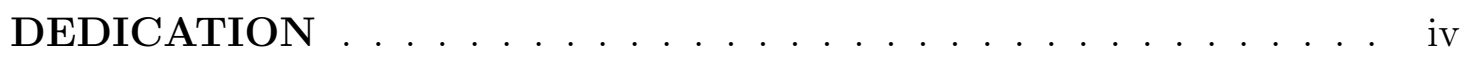

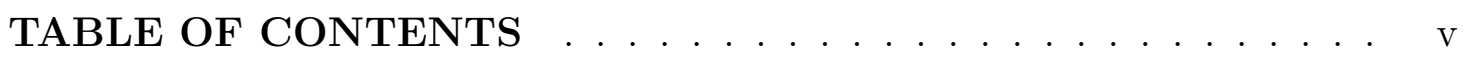

LIST OF TABLES . . . . . . . . . . . . . . . vii

LIST OF FIGURES . . . . . . . . . . . . . . . . . v viii

\section{CHAPTER}

1 Introduction and Background . . . . . . . . . . . . 1

1.1 Introduction . . . . . . . . . . . . . . . . 1

1.2 Graph Colorings . . . . . . . . . . . . . . . . . . . 4

List of References . . . . . . . . . . . . . . . . . . . . 9 9

$2(3,0,0)$-Colorability . . . . . . . . . . . . . . . 12

2.1 Introduction . . . . . . . . . . . . . . . . . 12

2.2 Definitions and Charges $\ldots \ldots \ldots \ldots \ldots$

2.3 Lemmas . . . . . . . . . . . . . . . . . . . . . . . . . . 14

2.4 Discharging Procedures . . . . . . . . . . . . . . . 20

2.5 The New Charges . . . . . . . . . . . . . . . . . 20

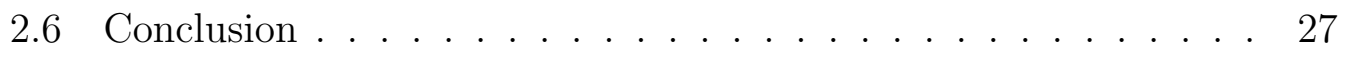

List of References . . . . . . . . . . . . . . . . . 27

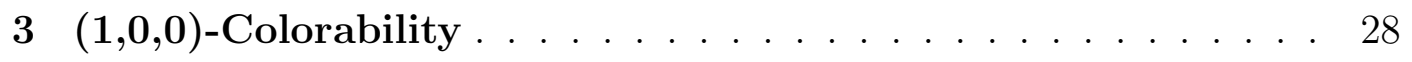




\section{Page}

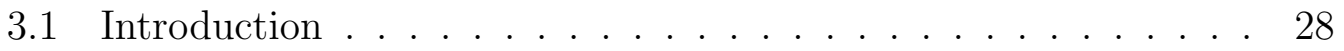

3.2 Definitions and Charges $\ldots \ldots \ldots \ldots$

3.3 Lemmas . . . . . . . . . . . . . . . . . . . . . . . 32

3.4 Discharging . . . . . . . . . . . . . . . . . 36

3.5 New Charges . . . . . . . . . . . . . . . . . . . . . 37

3.6 Conclusion . . . . . . . . . . . . . . . . . . . . . . . . 47

List of References . . . . . . . . . . . . . . 47

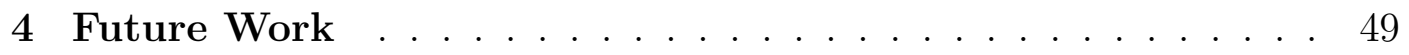

4.1 Planar Graphs with no 5- or 8-Cycles or Adjacent 3-Cycles . . . 49

4.2 Steinberg's Conjecture . . . . . . . . . . . . . . . . . 52

List of References . . . . . . . . . . . . . . . . . 5 52

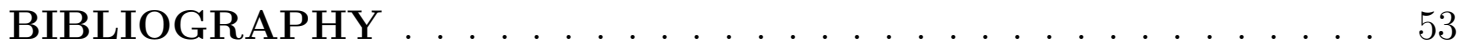




\section{LIST OF TABLES}

Table

Page

$2.1 \quad$ Forbidden Cases . . . . . . . . . . . . . . 23 


\section{LIST OF FIGURES}

$\begin{array}{lll}\text { Figure } & \text { Page }\end{array}$

$1.1 \quad$ A graph $G$ (which is planar) . . . . . . . . . . . 2

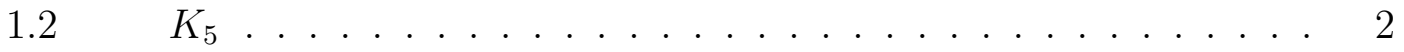

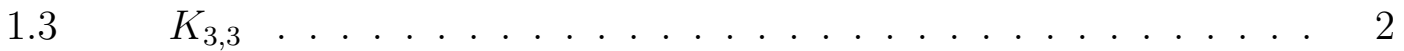

1.4 A 2-coloring of $\mathrm{G}$, with color set $\{1,2\} \ldots \ldots$

1.5 A $(2,1)$-coloring of $\mathrm{G}$, with color set $\{a, b\} \ldots \ldots$

$2.1 \quad f$, a pendent face of $v \ldots \ldots \ldots \ldots$

$2.2 \quad \mathrm{~A}(3,3,7)$-star-face, $f \ldots \ldots \ldots \ldots$

$2.3 \quad$ A full vertex $v$ with special vertex $u$. . . . . . . . . . 18

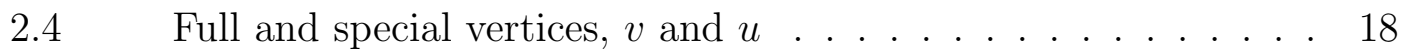

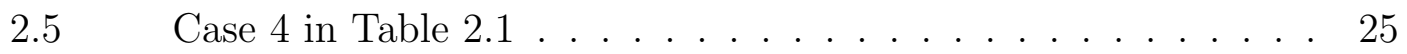

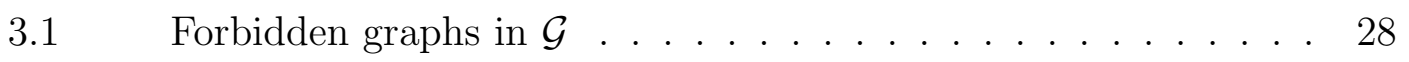

3.2 A $b_{8}$-face $f \ldots \ldots \ldots \ldots \ldots$

$3.3 \quad \mathrm{~A}$ bad 6 -face .............................. 30

$3.4 \quad$ A bad 8 -face . . . . . . . . . . . . . . . . 30

3.5 Two bad 9 -faces . . . . . . . . . . . . . . . 31

3.6 A $3^{*}$-face $f(j=6$ or 9$\left.)\right) \ldots \ldots . \ldots . \ldots . \ldots 31$

3.7 A vertex $u \in G(k)(j=8$ or 9$) \ldots \ldots . \ldots . \ldots 31$

3.8 Impossible configuration, as in Lemma $2 \ldots . . . . . . . .33$

Lemma 6 Configuration . . . . . . . . . . . . . . 39

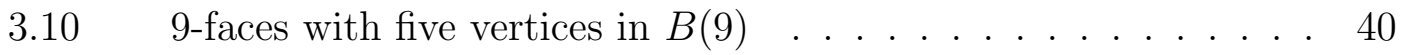

3.11 A 9-face with six vertices in $B(9) \ldots \ldots . . . . . . . .441$ 
3.12 An 8-face with five vertices in $B(8)$ (labeled $\left.v_{1}, v_{2}, \ldots, v_{5}\right) \ldots 46$

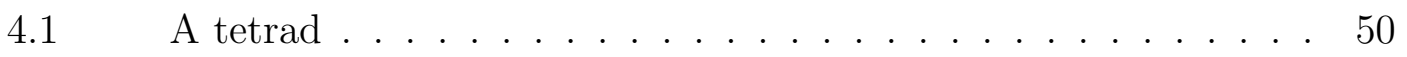

$4.2 \quad$ An internal face with pseudo-tetrad . . . . . . . . . . . 51

$4.3 \quad$ An internal 9-face with negative final charge . . . . . . . 51

$4.4 \quad$ An internal 10-face with negative final charge . . . . . . . . . 52 


\section{CHAPTER 1}

\section{Introduction and Background}

\subsection{Introduction}

Combinatorics, especially graph theory, is an ever-increasing field of research. New methods of approaching different types of problems are developed quite often in graph theory, leading to answers of long ago proposed questions as well as theorizing new conjectures.

Graph theory in general has many applications for various fields of study, most notably are computer science and computer engineering. Graphs are used for scheduling and networking problems as well as other areas. Since many real-life situations involve discrete objects, graph theory lends itself very well to these types of problems. Any type of map or network (say of pipes, people, or circuits) can be modeled using a graph.

In this paper, we deal exclusively with simple graphs. A simple graph $G=$ $(V, E)$ is a graph which consists of vertices $v \in V$ and edges $\{u, v\} \in E$, which are pairs of vertices, in which the edge set contains no duplicate pairs and no edge consists of only one vertex (ie, no edge has the form $\{v, v\}$ for $v \in V$ ). Graphs

can contain paths and cycles. A path is an ordered set of vertices $\left\{v_{1}, v_{2}, \ldots, v_{n}\right\}$ such that there exists an edge between each consecutive pair of vertices. A cycle is a path such that there exists an edge between $v_{1}$ and $v_{n}$. The set $\{b, c, e, d\}$ is a cycle in Figure 1.1. A chord is an edge connecting two nonconsecutive vertices in a cycle.

Several types of graphs are very useful in graph theory and are therefore given their own names. We mention just a few here. A complete graph, denoted $K_{n}$, is a graph on $n$ vertices such that every two vertices are connected. A bipartite graph is a graph whose vertex set can be divided into two mutually disjoint sets of vertices. 


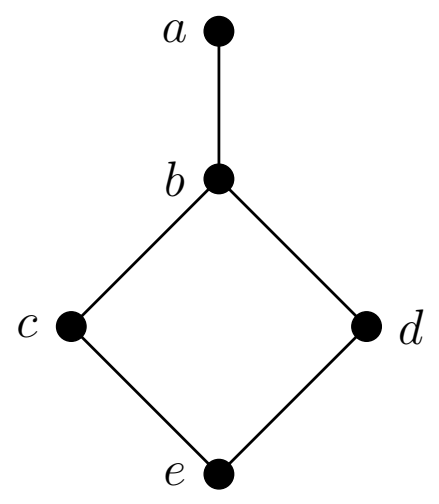

Figure 1.1. A graph $G$ (which is planar)

That is, there exist no edges between any two vertices in the same set. A complete bipartite graph, denoted $K_{n, m}$, is a bipartite graph with vertex sets $A$ and $B$, where $|A|=n$ and $|B|=m$, that contains every edge of the form $\left\{v_{a}, v_{b}\right\}$ where $v_{a} \in A$ and $v_{b} \in B$. See Figures 1.2 and 1.3 for examples of a complete graph and a complete bipartite graph.

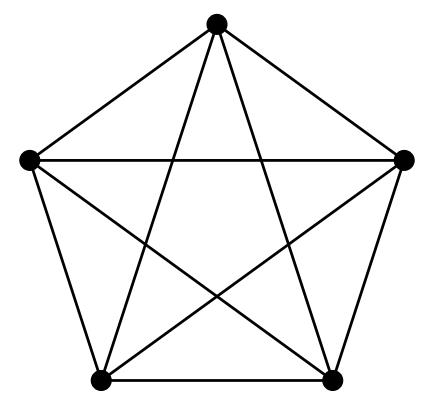

Figure 1.2. The complete graph $K_{5}$

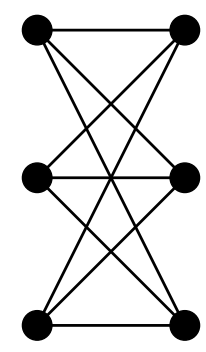

Figure 1.3. The complete bipartite graph $K_{3,3}$, which is non-planar

In this work, we deal primarily with planar graphs. A planar graph is a graph 
that can be drawn in the Euclidean plane in such a way that edges meet only at their endpoints. A plane graph (or a plane drawing of a planar graph) is a planar graph that is drawn with no edges crossing each other, except at their endpoints. It is known that a graph is planar if and only if it contains no $K_{5}$ or $K_{3,3}$ subdivisions. See Figure 1.1 for an example of a planar graph and Figure 1.3 for an example of a non-planar graph.

Besides vertices and edges, a planar graph also contains faces. A face is a region of the graph bounded by edges such that no edge goes through it. In Figure 1.1, the region bounded by the cycle $\{b, c, e, d\}$ is a face. The set of all faces of a graph is denoted by $F$. The unbounded or outer face of a graph is the face outside of the graph. In Figure 1.1, the region outside the cycle $\{b, c, e, d\}$ is the unbounded face. One type of planar graph is called an outerplanar graph. Euler gave a formula that shows the relationship between the faces, vertices, and edges of planar graphs. He proved that $|V|+|F|-|E|=2$ for any plane drawing of a planar graph.

Now we turn our attention to what mathematicians do with graphs. Graphs are very helpful when it comes to modeling many discrete things in every day life. Whenever one has a collection of discrete objects that interact in a certain way, or have certain shared characteristics, one can model it by a graph. For example, let the vertices be people in a town, and let the edges be pairs of people that know each other. This is a basic example of a social network graph. These types of graphs are currently being studied a lot, especially with ties to social media and the internet.

Graph theorists over the years have studied several aspects of graphs. Some look at how we can use graphs to model real life situations. Some try to find certain subgraphs in large graphs using computer aided software. Others work on 
characterizing graphs with a certain attribute. For example, what types of graphs have a Hamiltonian cycle? (A Hamiltonian cycle is a cycle that uses all the vertices of the graph.)

\subsection{Graph Colorings}

One of the characteristics we are concerned with is graph colorings. A proper coloring of a graph $G=(V, E)$ is a mapping $\phi: V \rightarrow S$ where $S$ is some set, called the color set, such that if $\{u, v\} \in E$, then $\phi(u) \neq \phi(v)$. Generally, we take $S$ to be a set of colors or numbers. A graph is said to be $\mathbf{k}$-colorable if it can be properly colored using $k$ colors. See Figure 1.4 for a 2-colorable graph. If $k$ is the smallest such number, we say the graph has chromatic number equal to $k$.

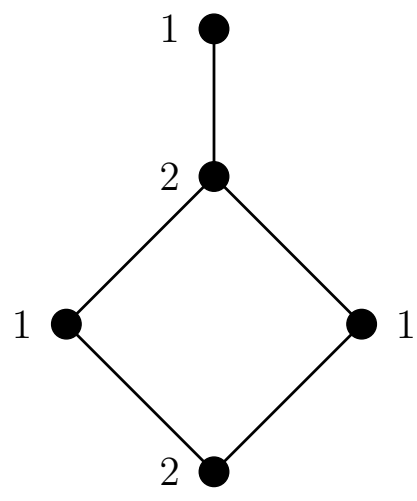

Figure 1.4. A 2-coloring of $\mathrm{G}$, with color set $\{1,2\}$

Work has been done on graph colorings for well over a century. Some mathematicians are concerned with only coloring planar graphs. However, there is a very famous graph coloring problem that deals with a non-planar graph. The HadwigerNelson problem was proposed in 1950 by E. Nelson. Let $G$ be the infinite graph whose vertex set is the set of all points in the Cartesian plane, and let two vertices be adjacent if and only if they are one unit distance apart. It has been shown that at least four colors are needed to properly color $G$, and that it can be colored using only seven. The Hadwiger-Nelson problem is to find the exact chromatic number 
of this graph. In over sixty years of study, it is still open.

The most famous coloring problem dealing with planar graphs is the Four Color Theorem, which was first postulated by Francis Guthrie in 1852 [1]. He proposed that one could color the countries in a map using only four colors such that no countries sharing a border were colored the same. If each country is represented by a vertex, and two vertices are connected by an edge if and only if the two countries share a border, then we can translate the map into a planar graph. The Four Color Theorem then says

Four Color Theorem. Every planar graph can be properly colored with at most four colors.

This is the best possible in the sense that the planar graph $K_{4}$ cannot be colored with less than four colors.

While trying to prove the Four Color Theorem, Heawood [2] proved a relaxation of it in 1890. He proved the Five Color Theorem:

Five Color Theorem. Every planar graph can be properly colored with at most five colors.

Despite constant study and analysis by a multitude of mathematicians, the Four Color Theorem remained unsolved for over a century. In 1976, Appel and Haken $[3,4]$ finally gave a very lengthy, computer aided proof of the theorem. To this day, mathematicians are still trying to find an elegant, non-computer generated proof.

Graph theorists also ask themselves when a graph can be properly colored with fewer than four colors. A graph is 1-colorable if and only if there are no edges in the graph. Determining when a graph is two-colorable is also quite easy. A graph is 2-colorable if and only if it is bipartite [5]. A graph is bipartite if and only if it contains no odd cycles. 
Deciding when a graph is 3-colorable, however, takes much more work. We know that all outerplanar graphs are 3-colorable [6], where an outerplanar graph is a planar graph which has a plane drawing such that every vertex is on the unbounded face. Grötzsch [7] proved the following interesting result about planar graphs in 1959 .

Grötzsch's Theorem. Every triangle-free planar graph is 3-colorable.

In 1963, Grünbaum [8] extended this and proved the following.

Grünbaum's Theorem. Every planar graph with not more than three triangles is 3-colorable.

We now come to a very famous conjecture, proposed by R. Steinberg in 1976 $[9]$.

Steinberg's Conjecture. Every planar graph without cycles of length 4 or 5 is 3-colorable.

A lot of work has been done towards this conjecture, but the conjecture itself remains unsolved. Erdős stated a relaxation of Steinberg's Conjecture in 1991. He proposed determining the smallest integer $k$ (if it exists) such that all planar graphs without cycles of length 4 through $k$ are 3-colorable. Much progress was made using this relaxation. In 1991, Abbott and Zhou [10] proved $k \leq 11$. In 1995, Sanders and Zhao [11], and independently Borodin [12], improved this result to $k \leq 9$. Then in 2002, Salavatipour [13] went further and showed $k \leq 8$. Lastly, Borodin, Glebov, Raspaud, and Salavatipour [14] showed $k \leq 7$ in 2005.

It was at this stage that people began looking at Steinberg's Conjecture from a new direction. A new type of coloring, a non-proper coloring, was introduced. Defective colorings (also called improper colorings or near colorings) are graph 
colorings where, in certain circumstances, adjacent vertices may be colored the same.

One type of defective coloring is a $(k, d)$ coloring. A graph is said to be $(k, d)$-colorable if it can be colored with $k$ colors in such a way that the maximum degree of the subgraph induced by each color class is at most $d$. The degree of a vertex is the number of vertices adjacent to it. By the subgraph induced by a color class, we mean the induced subgraph whose vertices are all the vertices colored by the same color. Many mathematicians found interesting results using this new coloring. Cowen, Cowen, and Woodall [15] proved that all planar graphs are (3,2)-colorable. In 2008, Xu [16] showed that all planar graphs without cycles of length five or adjacent triangles are $(3,1)$-colorable. See Figure 1.5 for a graph that is $(2,1)$-colorable.

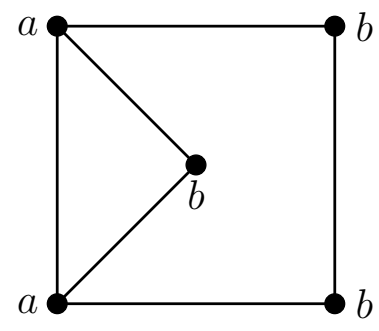

Figure 1.5. A $(2,1)$-coloring of $\mathrm{G}$, with color set $\{a, b\}$

Graph theorists then combined defective colorings with list colorings of graphs. An $L$-list coloring of a graph $G$ is a coloring of the vertices of $G$ such that each color comes from a prescribed list of colors $L(v)$ for each vertex. $G$ is $k$-choosable or $k$-list colorable if $G$ can be properly colored using colors from any list coloring $L(v)$ with size $k$ for each vertex. A graph $G$ is then $(k, d)$-choosable if the vertices of $G$ can be colored with colors from any list $L(v)$ of size $k$ for each vertex such that the degree of each induced color class is at most d. In 1999, Eaton and Hull [17], and independently Škrekovski [18], proved every planar graph is $(3,2)$-choosable, 
Eaton and Hull also proved every outerplanar graph is in fact $(2,2)$-choosable. In 2001, Lih, Song, Wang, and Zhang [19] proved that every planar graph without cycles of length 4 or 5 are $(3,1)$-choosable.

Chang, Havet, Montassier, and Raspaud [20] looked at another variation of defective colorings in 2012. They decided to look at colorings where the maximum degree of the color class depended on the color. A graph is said to be $\left(s_{1}, s_{2}, s_{3}\right)$ colorable if there exists a coloring of the graph with three colors $(1,2,3)$ such that the induced subgraph on the $i^{t h}$ color class has degree at most $s_{i}$ for $i=1,2,3$. Ultimately, the goal is to show that all planar graphs without cycles of length 4 or 5 are $(0,0,0)$-colorable, thus proving Steinberg's Conjecture. We focus on making each $s_{i}$ as small as possible, for $i=1,2,3$. Chang, et al. showed that all planar graphs without cycles of length 4 or 5 are $(4,0,0)$ - and $(2,1,0)$-colorable.

Depending on the application, either coloring of a graph could be useful. Having two perfect colors and one color that is defective is pretty nice if the second two colors are more important to the application. A color class of degree four can get ugly fast. For example, a $(4,0,0)$-coloring of a planar graph could leave a $K_{1,4}$ monochromatic. The $(2,1,0)$-coloring is better in that the degrees are smaller (you may only have a monochromatic path or cycle in the first color or a monochromatic matching in the second), but now two colors are defective. Defective colorings are a give and take, we might be able to decrease the overall defective degree, but then the number of defective colors can increases as a result. As an example of a defective coloring, we can think of scheduling final exams. The vertices are the courses and they are connected by an edge if the final exam occurs at the same time. We use the colors to represent which classroom we will use for each final. Suppose one of the classrooms is very large and has enough room for two final exams to occur at once. This means we could color two adjacent vertices 
by the color corresponding to this room (but not, say, a triangle). This would give us a $(1,0,0)$-coloring, if we only had three rooms available.

Hill and $\mathrm{Yu}$ [21], and independently Xu, Miao, and Wang [22], improved Chang, et al.'s second result, proving that every planar graph without cycles of length 4 or 5 is actually $(1,1,0)$-colorable. Hill and $\mathrm{Yu}, \mathrm{Xu}$ and Wang, and this author also independently improved upon Chang, et al.'s first result, showing that all such planar graphs are $(3,0,0)$-colorable. The three sets of papers were combined into one submission [23]. The version written by this author is shown in Chapter 2.

Much more work has been done using this idea of defective colorings in the past few years. Wang, Jin, and Kang [24] showed that all planar graphs without cycles of length 4 through 6 are $(1,0,0)$-colorable. Wang and $\mathrm{Xu}$ [25] proved all planar graphs without cycles of length 4 or 6 are $(2,0,0)$-colorable, and they also showed in another paper that all planar graphs without 4- or 6-cycles, and all planar graphs without 4- or 7-cycles, are (1,1,0)-colorable [26]. Bu and Fu [27] also showed that all planar graphs without cycles of length 4 or 6 are $(1,1,0)$-colorable. Wang and Yang [28] proved all planar graphs without cycles of length 4, 5, or 9 are $(1,0,0)$-colorable. Chapter 3 proves that all planar graphs without cycles of length 4 or 5 and without a few types of cycles of length 8 are $(1,0,0)$-colorable.

\section{List of References}

[1] P. Maritz and S. Mouton, "Francis Guthrie: A colourful life," The Mathematical Intelligencer, vol. 34, pp. 67-75, 2012.

[2] P. Heawood, "Map colour theorem," Quarterly Journal of Pure and Applied Mathematics, pp. 332-338, 1890.

[3] K. Appel and W. Haken, "Every planar map is four colorable. Part I. Discharging," Illinois Journal of Mathematics, pp. 429-490, 1977.

[4] K. Appel and W. Haken, "Every planar map is four colorable. Part II. Reducibility," Illinois Journal of Mathematics, pp. 491-567, 1977. 
[5] D. West, Introduction to Graph Theory, 2nd ed. Prentice Hall, 2001, ch. 5, p. 192.

[6] A. Proskurowski and M. Syslo, "Efficient vertex-and edge-coloring of outerplanar graphs," Society for Industrial and Applied Mathematics Journal on Algebraic Discrete Methods, vol. 7, no. 1, pp. 131-136, 1986.

[7] H. Grötzsch, "Ein dreifarbensatz für dreikreisfreie netze auf der kugel,," Math.-Naturwiss. Reihe 8, pp. 109-120, 1959, in German.

[8] B. Grünbaum, "Grötzsch's theorem on 3-colorings," Michigan Mathematics Journal, pp. 303-310, 1963.

[9] R. Steinberg, "The state of the three color problem, quo vadis, graph theory?" Annals of Discrete Mathematics, pp. 211-248, 1993.

[10] H. Abbott and B. Zhou, "On small faces in 4-critical graphs," Ars Combinatoria, pp. 203-207, 1991.

[11] D. Sanders and Y. Zhao, "A note on the three color problem," Graphs and Combinatorics, pp. 91-94, 1995.

[12] O. Borodin, "Structural properties of plane graphs without adjacent triangles and an application to 3-colorings," Journal of Graph Theory, vol. 21, pp. 183-186, 1996.

[13] M. Salavatipour, "The three color problem for planar graphs," Department of Computer Science, University of Toronto, Toronto, Ontario, Canada, Tech. Rep., July 2002.

[14] O. Borodin, A. Glebov, A. Raspaud, and M. Salavatipour, "Planar graphs without cycles of length from 4 to 7 are 3-colorable," Journal of Combinatorial Theory, pp. 303-311, 2005.

[15] L. Cowen, R. Cowen, and D. Woodall, "Defective colorings of graphs in surfaces: partitions into subgraphs of bounded valency," Journal of Graph Theory, pp. 187-195, 1986.

[16] B. Xu, "On $(3,1)^{*}$-coloring of plane graphs," Society for Industrial and Applied Mathematics Journal of Discrete Mathematics, vol. 1, pp. 205-220, 2008/09.

[17] N. Eaton and T. Hull, "Defective list colorings of planar graphs," Bulletin of the Institute of Combinatorics and its Applications, pp. 79-87, 1999.

[18] R. Škrekovski, "List improper coloring of planar graphs," Combinatorics Probability and Computing, pp. 293-299, 1999.

[19] K. Lih, Z. Song, W. Wang, and K. Zhang, "A note on list improper coloring planar graphs," Applied Mathematics Letters, pp. 269-273, 2001. 
[20] G. Chang, F. Havet, M. Montassier, and A. Raspaud, "Steinberg's conjecture and near colorings," manuscript.

[21] O. Hill and G. Yu. Arxiv. "A relaxation of Steinberg's conjecture." [Online]. Available: arxiv.org/abs/1208.3395

[22] L. Xu, Z. Miao, and Y. Wang, "Every planar graph with cycles of length neither 4 nor 5 is $(1,1,0)$-colorable," Journal of Combinatorial Optimization, DOI 10.10071s10878-012-9586-4.

[23] O. Hill, D. Smith, Y. Wang, L. Xu, and G. Yu, "Planar graphs without cycles of length 4 or 5 are $(3,0,0)$-colorable," Discrete Mathematics, vol. 313, pp. $2312-2317,2013$.

[24] Y. Wang, L. Jin, and Y. Kang, "Planar graphs without cycles of length from 4 to 6 are (1, 0, 0)-colorable," Scientia Sinica Mathematica, vol. 43, pp. 11451164, 2013, in Chinese.

[25] Y. Wang and J. Xu, "Planar graphs with cycles of length neither 4 nor 6 are (2,0,0)-colorable," Information Processing Letters, vol. 113, pp. 659-663, 2013.

[26] Y. Wang and J. Xu, "Improper colorability of planar graphs without prescribed short cycles," Discrete Mathematics, vol. 322, pp. 5-14, 2014.

[27] Y. Bu and C. Fu, "(1,1,0)-coloring of planar graphs without cycles of length 4 and 6," Discrete Mathematics, vol. 313, pp. 2737 - 2741, 2013.

[28] Y. Wang and Y. Yang, "(1,0,0)-colorability of planar graphs without cycles of length 4, 5, or 9," 2012, submitted. 


\section{CHAPTER 2}

\section{$(3,0,0)-$ Colorability}

\subsection{Introduction}

In this study, we deal only with finite, simple graphs. We consider $\mathcal{F}$, the family of planar graphs without 4- and 5-cycles. Chang et al. [1] proved that every graph in $\mathcal{F}$ is $(2,1,0)$-colorable and $(4,0,0)$-colorable. Using similar techniques, we improve on their second result:

Theorem 1. Every graph in $F$ is $(3,0,0)$-colorable.

We begin with some definitions and placing charges on the faces and vertices of a minimal counterexample. Then we introduce some lemmas dealing with necessary configurations as well as forbidden subgraphs in this counterexample. Lastly, we redistribute the charges and reach a contradiction, proving this counterexample cannot exist, which in turn proves Theorem 1.

\subsection{Definitions and Charges}

For a general $\left(s_{1}, s_{2}, s_{3}\right)$-coloring of a graph, we say a vertex is nicely colored (by $i$ ) if it, and at most $\max \left\{0, s_{i}-1\right\}$ of its neighbors, are colored by $i$. We say a vertex is properly colored if it is colored by a color not used on its neighbors.

We denote a vertex of degree $k$ as a $k$-vertex, a vertex of degree less than or equal to $k$ as a $k^{-}$-vertex, and a vertex of degree greater than or equal to $k$ as a $k^{+}$-vertex. Similarly, a $k$-face is a face with $k$ edges.

If $v$ is a vertex on a face, $f$, we say that $f$ is an incident face of $v$. If $v$ is not incident to $f$, but is adjacent to a 3-vertex $u$ which is incident to $f$, then $f$ is a pendent face of $v$, and $v$ is the outer neighbor of $u$. See Figure 2.1 for an example. 


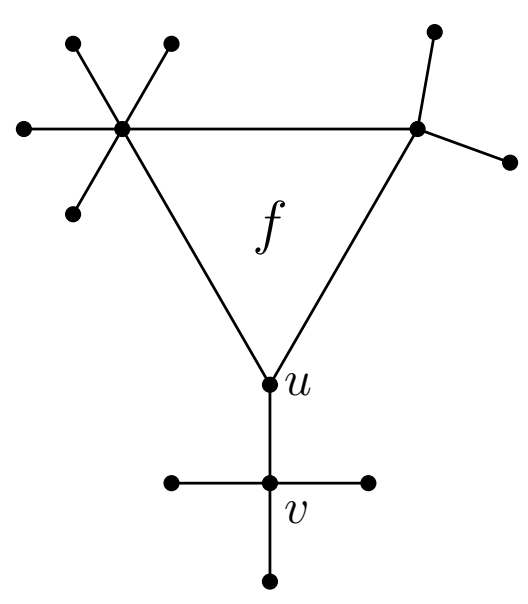

Figure 2.1. $f$, a pendent face of $v$ (which is an outer neighbor of $u$ )

A $\left(d_{1}, d_{2}, d_{3}\right)$-face is a 3 -face with vertices $v_{1}, v_{2}, v_{3}$ having degrees $d_{1}, d_{2}, d_{3}$, respectively. Similarly, $d_{i}$ can be replaced with $d_{i}^{+}$, meaning $d\left(v_{i}\right) \geq d_{i}$ or $d_{i}^{-}$, meaning $d\left(v_{i}\right) \leq d_{i}$, where $d\left(v_{i}\right)$ is the degree of $v_{i}$.

Let $G \in \mathcal{F}$ be a minimal graph (on number of vertices) such that $G$ is not $(3,0,0)$-colorable. That is, $G$ is a planar graph without cycles of length 4 or 5 and is not $(3,0,0)$-colorable, but $G-v$ for any $v \in V(G)$ is $(3,0,0)$-colorable. Notice that $G$ is connected. We will assign each face and vertex of $G$ a charge, denoted by $\operatorname{ch}(x)$. Let $F$ denote the set of faces in $G$.

We start with $\operatorname{ch}(v)=2 d(v)-6$ for all $v \in V=V(G)$ and $\operatorname{ch}(f)=d(f)-6$ for all $f \in F$, where $d(f)$ is the number of vertices incident to $f$, counting multiplicity. We have

$$
\begin{aligned}
\sum_{v \in V} \operatorname{ch}(v)+\sum_{f \in F} \operatorname{ch}(f) & =\sum_{v \in V}(2 d(v)-6)+\sum_{f \in F}(d(f)-6) \\
& =4|E(G)|-6|V(G)|+2|E(G)|-6|F| \\
& =-6(|F|+|V(G)|-|E(G)|) \\
& =-6(2) \\
& =-12
\end{aligned}
$$

by Euler's Formula.

After establishing some properties of $G$, we will redistribute the charges to the 
faces and vertices, while neither creating nor destroying any charges. Afterwards, we will show that the new charge of each face and vertex is nonnegative, reaching a contradiction to the fact that they sum to -12 . Therefore, this graph $G$ cannot exist, thus proving all graphs without cycles of length 4 or 5 are $(3,0,0)$-colorable.

\subsection{Lemmas}

First, we note that $d(v) \geq 3$ for all $v \in V$ and no two 3-faces may share an edge. The following two lemmas come directly from Chang et al. [1]. We include the proofs for completeness. Some results based on these lemmas are then given.

Lemma 1. (Chang, Havet, Montassier, Raspaud). If a k-vertex is incident to $\alpha$ 3-faces and has $\beta$ pendent 3-faces, then $2 \alpha+\beta \leq k$.

Proof. Assume $2 \alpha+\beta>k$ for some $k$-vertex $v$. Let $x_{1}, x_{2}, \ldots, x_{2 \alpha}$ be the neighbors of $v$ on the $\alpha$ 3-faces. Let $y_{1}, y_{2}, \ldots, y_{\beta}$ be the neighbors of $v$ incident to the $\beta$ 3 -faces. Since $2 \alpha+\beta>k$, at least one $y_{i}$ must be an $x_{j}$ for some $i$ and $j$. We then have $d\left(y_{i}\right) \geq 4$, since the two 3 -faces cannot share an edge. This contradicts the definition of a pendent 3-face. Hence, we must have $2 \alpha+\beta \leq k$.

Lemma 2. (Chang, Havet, Montassier, Raspaud). The three neighbors $x_{1}, x_{2}, x_{3}$ of a 3-vertex $v$ of $G$ use different colors in a $\left(s_{1}, s_{2}, s_{3}\right)$-coloring of $G-v$. Moreover, assume $x_{i}$ is colored by $i$, we have $d\left(x_{i}\right) \geq s_{i}+3$ for $1 \leq i \leq 3$. Furthermore, if $s_{i}>0$ and $x_{i}$ is adjacent to $x_{j}$, then either $d\left(x_{i}\right)>s_{i}+3$ or $d\left(x_{j}\right)>s_{j}+3$.

Proof. If $x_{1}, x_{2}, x_{3}$ do not use three distinct colors, then we can properly color $v$, a contradiction. Hence, without loss of generality, we can assume that $x_{i}$ is colored by $i$ for $1 \leq i \leq 3$.

Suppose for a contradiction that some $d\left(x_{i}\right) \leq s_{i}+2$. Then $s_{i} \geq 1$ as $d\left(x_{i}\right) \geq 3$. If $x_{i}$ is nicely colored by $i$, then we color $v$ by $i$, and this extends the coloring to $G$, a contradiction. Hence, $x_{i}$ has $s_{i}$ neighbors colored by $i$. Since $x_{i}$ has an uncolored 
neighbor $v$, there is at least one color different from $i$ not used by $x_{i}$ 's neighbors. We then color $v$ by $i$ and recolor $x_{i}$ by the unused color. This extends the coloring to $G$, a contradiction.

Suppose for another contradiction that $x_{i}$ is adjacent to $x_{j}$, but $d\left(x_{i}\right)=s_{i}+3$ and $d\left(x_{j}\right)=s_{j}+3$ where $s_{i} \geq 1$. Let $k$ be the color distinct from $i$ and $j$. Since $G$ has no 4 -cycle, $x_{k}$ is not adjacent to $x_{i}$ or $x_{j}$. As above, $x_{i}$ (resp. $x_{j}$ ) has $s_{i}$ (resp. $s_{j}$ ) neighbors colored by $i$ (resp. $j$ ) and another colored neighbor $x_{i}^{\prime}\left(\operatorname{resp} . x_{j}^{\prime}\right)$ other than $x_{j}\left(\operatorname{resp} . x_{i}\right)$. If $x_{i}^{\prime}$ is colored by $j$, then we may color $v$ by $i$ and recolor $x_{i}$ by $k$ to get a $\left(s_{1}, s_{2}, s_{3}\right)$-coloring of $G$, a contradiction. Hence, $x_{i}^{\prime}$ is colored by $k$. Similarly, $x_{j}^{\prime}$ is also colored by $k$. Then we may color $v$ by $i$, recolor $x_{i}$ by $j$, and recolor $x_{j}$ by $i$ to get a $\left(s_{1}, s_{2}, s_{3}\right)$-coloring of $G$, again a contradiction. Hence, $d\left(x_{i}\right)>s_{i}+3$ or $d\left(x_{j}\right)>s_{j}+3$.

Lemma 3. The outer neighbor of every 3 -vertex incident to a $\left(3,3,6^{-}\right)$-face has degree at least six.

Proof. Assume $u v w$ is the $\left(3,3,6^{-}\right)$-face, that is $d(u)=3, d(v)=3$, and $d(w) \leq 6$. Let $v$ 's outer neighbor be called $x$. By Lemma 2, we have the degrees of the neighbors of $v$ are at least 3,3 , and 6 . So if $d(w) \leq 5$, then the degree of $x$ must be at least six. So assume $d(w)=6$ and $d(x) \leq 5$. Let $c$ be a $(3,0,0)$-coloring of $G-v$. By Lemma 2, we have $c(w)=1$, since it is the only neighbor of $v$ with degree at least $s_{1}+3=6$. But since $u w \in E(G)$, we need $d(w)>6$ or $d(u)>3$, a contradiction. So we must have $d(x) \geq 6$. The same is true for the outer neighbor of $u$ and $w$ if $d(w)=3$.

These first few lemmas dealt with configurations that must be in $G$. The next two lemmas look at some forbidden subgraphs of $G$.

Lemma 4. Suppose a k-vertex $v(k=7,8)$ is incident to $(k-5)(3,3, k)$-faces. Then, if $k=7, v$ cannot also be incident to a $(3, j, 7)$-face with $j \in\{3,4\}$ and 
have its seventh neighbor be a 3-vertex, and if $k=8, v$ cannot also be incident to $a(3, j, 8)$-face with $j \in\{3,4\}$.

Proof. Assume for a contradiction that $v$ is a such a $k$-vertex. Let $N^{\prime}(v)$ denote the neighbors of $v$ which have degree equal to 3 .

Let $c$ be a $(3,0,0)$-coloring of $G \backslash\left\{v, N^{\prime}(v)\right\}$. Extend this coloring to all $u \in N^{\prime}(v)$ such that each $u$ is properly colored. This is possible as each $u \in N^{\prime}(v)$ has at most two neighbors already colored.

At most four of the neighbors of $v$ are colored 1 (one in each 3-face if $k=8$ and one in each 3 -face plus the vertex not incident to one of the 3-faces if $k=7$ ). If there are three or less neighbors colored 1 , we can color $v$ by 1 (since each vertex colored 1 has at most two other neighbors colored 1), and thus extend the coloring to a $(3,0,0)$-coloring of $G$, a contradiction.

Therefore, we must have exactly four neighbors colored 1 . There are $(k-4)$ other neighbors not colored 1. If they are all colored the same color, then we can color $v$ the third color, and we reach a contradiction.

Hence, these other neighbors must use both colors 2 and 3 . We break this into two cases:

Case 1: Each vertex colored 2 is incident to one of the $(3,3, k)$-faces incident to $v$.

Let one such vertex be $x$. Label the neighbor of $x$ incident to the same 3-face as $u$ (note that $c(u)=1$ ), and label the outer neighbor of $x$ by $w$. If $c(w)=1$, we recolor $x$ by 3 . If $c(w)=3$, we recolor $x$ by 1 . Since the degree of $u$ is 3 , we still have a $(3,0,0)$-coloring of $G-v$. We can do the same to the other vertices colored 2 , if they exist. Now all the neighbors of $v$ use only two colors ( 1 and 3 ), so we can color $v$ by 2 , a contradiction.

Case 2: A vertex colored 2 is incident to the $(3, j, k)$-face with $j=4$.

We re-color the vertices colored 3 in the same way as above, leaving all the 
neighbors of $v$ colored either 1 or 2 . We can then color $v$ by 3 , a contradiction. Thus, none of these configurations can exist in $G$.

We introduce a specific kind of face and pair of adjacent vertices next, which we use in the following lemma. For $j, k \geq 3$, let $f$ be a $(3, j, k)$-face where the degree of the outer neighbor of each incident 3-vertex is less than six. Then $f$ is called a $(3, j, k)$-star-face. See Figure 2.2 . Let $v$ be a $k$-vertex $(7 \leq k \leq 8)$ that is incident to $(k-5)(3,3, k)$-star-faces and one $(3, j, k)$-star-face with $j \geq 7$. In addition, let $v$ have a pendent 3 -face if $k=7$. We call such a vertex a full vertex. We call the unique neighbor of a full vertex with degree at least 7 a special vertex. Refer to Figure 2.3 for an example.

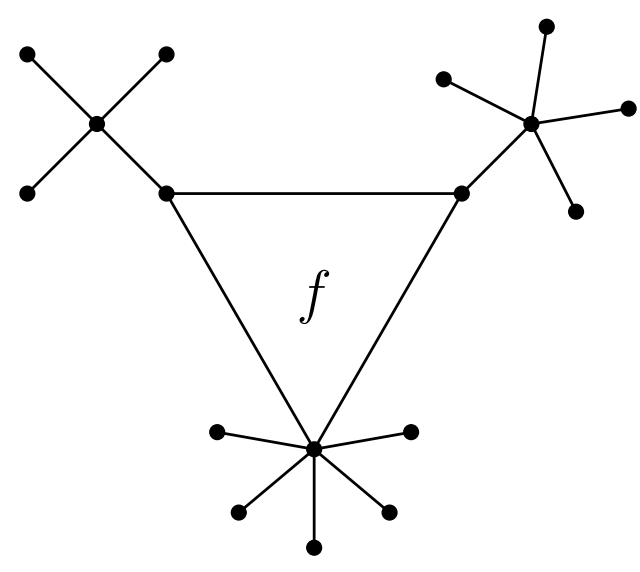

Figure 2.2. A $(3,3,7)$-star-face, $f$

Lemma 5. Let $v$ be a $k$-vertex with $7 \leq k \leq 8$. The vertex $v$ cannot be both a special vertex and a full vertex.

Proof. Suppose, for a contradiction, that $v$ is both a special and a full vertex. Since $v$ is full, it must be adjacent to a special vertex, call it $u$. Since $v$ is special, it must be adjacent to a full vertex of degree either 7 or 8 , and by uniqueness, this vertex must also be $u$. So $v$ and $u$ are both special and full. See Figure 2.4 for an example with $v$ being a 7 -vertex and $u$ being an 8-vertex. 


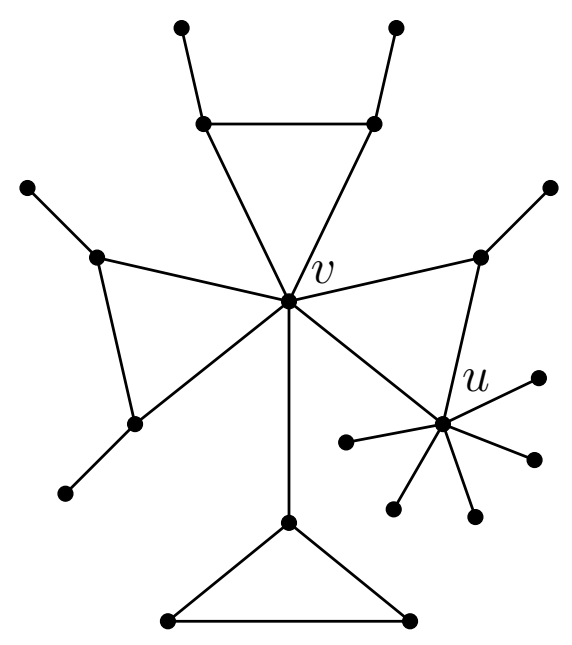

Figure 2.3. A full vertex $v$ with special vertex $u$

Let $c$ be a $(3,0,0)$-coloring of $G \backslash\left\{v, u, N^{\prime}(v), N^{\prime}(u)\right\}$ where $N^{\prime}(v)$ (respectively, $N^{\prime}(u)$ ) denotes the set of neighbors of $v$ (resp $u$ ) having degree three. Let the vertex incident to both $u$ and $v$ be called $w$ and let its outer neighbor be called $z$. We extend this $(3,0,0)$-coloring to $\left\{N^{\prime}(v), N^{\prime}(u)\right\} \backslash\{u, v, w\}$ in such a way as to properly color these vertices. This is possible as every vertex being properly colored has at most two neighbors previously colored.

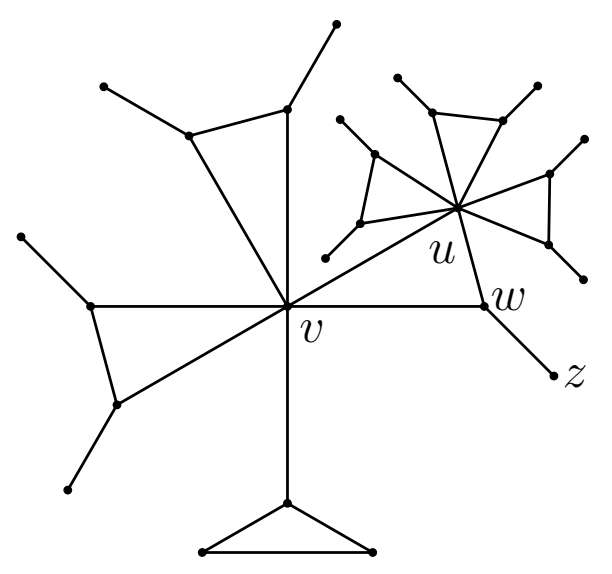

Figure 2.4. Full and special vertices $v$ and $u$

Note that $v$ and $u$ each have at most three neighbors colored 1 . Denote the set of neighbors of $v$ colored 1 by $M$ and the set of neighbors of $u$ colored 1 by $N$. We break this into three cases. 
Case 1: $|M| \leq 2$ and $|N| \leq 2$.

We can color both $v$ and $u$ by 1 , and then properly color $w$, thus extending the coloring to a $(3,0,0)$-coloring of $G$, a contradiction.

Case 2: The size of one of these sets is 3 , and the other is at most 2 .

Without loss of generality, we will assume $|N|=3$ and $|M| \leq 2$. If the colored neighbors of $u$ that are not colored 1 use the same color, then we can color $u$ by the third color and color $v$ by 1 . If $c(z)=1$, we can properly color $w$. If $c(z) \neq 1$, we can color $w$ by 1 (which is still legal, as $v$ previously had at most two neighbors colored by 1$)$, thus giving us a $(3,0,0)$-coloring of $G$.

Hence, the colored neighbors of $u$ must use all three colors. There is a color used exactly once on the neighbors of $u$, say 2 . Let that vertex be called $y$, and let its outer neighbor be denoted by $s$. We can recolor $y$ by $\{1,3\} \backslash\{c(s)\}$, color $u$ by $2, v$ by 1 , and color $w$ as above, either 1 or 3 . We now have a legal $(3,0,0)$-coloring of $G$, a contradiction.

Case 3: $|M|=|N|=3$.

Let $r$ be a neighbor of $u$ colored by 2 , if any exist. If $r$ 's outer neighbor is colored by 3, we may recolor $r$ by 1 (this is legal since the third neighbor of $r$, other than its outer neighbor and $u$, has degree 3 ). If its outer neighbor is colored by 1 , we may recolor $r$ by 3 . We can now color $u$ by 2 .

We can recolor any neighbor of $v$ colored by 3 in this same manner, so that it is now colored by either 1 or 2 . Color $v$ by 3 . All that is left to color is $w$.

If $c(z) \neq 1$, we can color $w$ by 1 , thus extending the coloring to a $(3,0,0)$ coloring of $G$. Hence, we must have $c(z)=1$. If $z$ is nicely colored, we can still color $w$ by 1 and have a $(3,0,0)$-coloring of $G$, so $z$ must have three neighbors colored 1. If we could recolor $z$ to either 2 or 3 , then we could properly color $w$ by 1 and have a legal coloring. Thus, $z$ must have a neighbor colored 2 and a neighbor 
colored 3 , but then $d(z) \geq 6$, a contradiction to the fact that $w$ is in a star-face.

Hence, none of these configurations can exist in $G$.

\subsection{Discharging Procedures}

We redistribute the charges in the following manner:

A1: A 4-vertex gives one to every incident 3-face.

A2: A 5-vertex gives two to every incident 3-face.

A3: A 6-vertex gives two to every incident 3-face.

A4: An $11^{+}$-vertex gives three to every incident 3 -face.

A5: A $6^{+}$-vertex gives one to every pendent 3 -face.

Let $v$ be a $k$-vertex with $7 \leq k \leq 10$.

B1: $v$ gives three to every incident $(3,3, k)$-star-face.

B2: $v$ gives two to every incident $(3,4, k)$-face and $(3,3, k)$-non-star-face.

Let $7 \leq j \leq 10$.

C1: If $v$ is a special vertex, then it gives two to its incident $(3, j, k)$-star-face incident to the full vertex.

C2: If $v$ is a full vertex, then $v$ gives one to its incident $(3, j, k)$-star-face.

C3: If $v$ is neither a special vertex nor a full vertex, then it gives $\frac{3}{2}$ to every incident $(3, j, k)$-star-face.

D1: $v$ gives one to every remaining incident 3 -face.

\subsection{The New Charges}

Let $c h^{*}(x)$ denote the new charge of the face or vertex. We show each face and vertex of $G$ now has a nonnegative charge. Recall that originally, $\operatorname{ch}(v)=2 d(v)-6$ and $\operatorname{ch}(f)=d(f)-6$.

First we consider $f$, a 3 -face in $G$.

If $f=(3,3,3)$ : Each 3-vertex has an outer neighbor with degree at least 6 by 
Lemma 3. So by A5, we have $c h^{*}(f)=c h(f)+1+1+1=d(f)-6+3=0$.

If $f=(3,3, k)$ with $4 \leq k \leq 6$ : Each 3 -vertex has an outer neighbor with degree at least 6 by Lemma 3 , and thus gives one to $f$ by A5. The $k$-vertex gives at least one to $f$ by either $\mathrm{A} 1, \mathrm{~A} 2$, or $\mathrm{A} 3$, depending on the value of $k$. We have $\operatorname{ch}^{*}(f) \geq \operatorname{ch}(f)+1+1+1=d(f)-6+3=0$.

If $f=(3,3, k)$ with $7 \leq k \leq 10$ : We break this into two cases.

Case 1: $f$ is a $(3,3, k)$-star-face.

By B1, the $k$-vertex gives three to $f$, and we have $c h^{*}(f)=\operatorname{ch}(f)+3=$ $d(f)-6+3=0$.

Case 2: $f$ is a $(3,3, k)$-non-star-face.

At least one of the 3-vertices has an outer neighbor of degree at least six, and therefore gives one to $f$ by A5. By B2, the $k$-vertex gives two to $f$ and we have $c h^{*}(f) \geq \operatorname{ch}(f)+2+1=d(f)-6+3=0$.

If $f=(3,3, k)$ with $k \geq 11$ : By A4, the $k$-vertex gives three to $f$ and we have $\operatorname{ch}^{*}(f) \geq \operatorname{ch}(f)+3=d(f)-6+3=0$.

If $f=(3,4,4)$ : By Lemma 2 , we know the degrees of the neighbors of a 3vertex must be at least $3,3,6$, so the outer neighbor of the 3 -vertex in $f$ must have degree at least six, and thus gives one to $f$ by A5. Each 4-vertex gives one to $f$ by $\mathrm{A} 1$, and so we have $c h^{*}(f)=\operatorname{ch}(f)+1+1+1=d(f)-6+3=0$.

If $f=(3,4,5)$ : By Lemma 2 , the degree of the outer neighbor of the 3 -vertex is at least 6 and gives one to $f$ by A5. The 4 -vertex gives one to $f$ and the 5 -vertex gives two to $f$, and we have $c h^{*}(f)=\operatorname{ch}(f)+1+1+2=d(f)-6+4=1>0$.

If $f=\left(3,4,6^{+}\right)$: By $\mathrm{A} 1$, the 4 -vertex gives one to $f$, and by either $\mathrm{A} 3, \mathrm{~A} 4$, or $\mathrm{B} 2$, the $6^{+}$-vertex gives at least two to $f$, and we get $c h^{*}(f) \geq \operatorname{ch}(f)+1+2=$ $d(f)-6+3=0$.

If $f=(3,5,5)$ : By Lemma 2 , the degree of the outer neighbor of the 3 -vertex 
is at least 6 and gives one to $f$ by A5. Each of the 5-vertices give two to $f$ by A2, and so we have $c h^{*}(f)=c h(f)+1+2+2=d(f)-6+5=2>0$.

If $f=\left(3, k, 6^{+}\right)$with $k=5,6$ : By A2 or A3, the $k$-vertex gives two to $f$, and by either $\mathrm{A} 3, \mathrm{~A} 4$, or $\mathrm{D} 1$, the $6^{+}$-vertex gives at least one to $f$, so we have $c h^{*}(f) \geq \operatorname{ch}(f)+2+1=d(f)-6+3=0$.

If $f=(3, j, k)$ with $7 \leq j, k \leq 10$ : We break this into three cases.

Case 1: $f$ is a $(3, j, k)$-star-face not incident to a special vertex.

By definition, $f$ cannot be incident to a full vertex, either. So by C3, the $j$-vertex and the $k$-vertex each give $\frac{3}{2}$ to $f$, and we have $\operatorname{ch}^{*}(f)=\operatorname{ch}(f)+\frac{3}{2}+\frac{3}{2}=$ $d(f)-6+3=0$.

Case 2: $f$ is a $(3, j, k)$-star-face incident to a special vertex.

By $\mathrm{C} 1$, the special vertex gives two to $f$, and by $\mathrm{C} 2$, the full vertex that $v$ must be incident to gives one to $f$, giving $c^{*}(f)=c h(f)+2+1=d(f)-6+3=0$. Case 3: $f$ is a $(3, j, k)$-non-star-face.

The outer neighbor of the 3-vertex gives one to $f$ by A5. The $j$-vertex and the $k$-vertex each give one to $f$ by $\mathrm{D} 1$, and we have $\operatorname{ch}^{*}(f)=\operatorname{ch}(f)+1+1+1=$ $d(f)-6+3=0$.

If $f=\left(3,7^{+}, 11^{+}\right)$: By A4, the $11^{+}$-vertex gives three to $f$, so we have $c h^{*}(f) \geq$ $\operatorname{ch}(f)+3=d(f)-6+3=0$.

If $f=\left(4^{+}, 4^{+}, 4^{+}\right)$: Every vertex gives at least one to $f$ by A1, A2, A3, A4, and D1, so we have $c h^{*}(f) \geq c h(f)+1+1+1=d(f)-6+3=0$.

No 4- or 5-faces exist in $G$.

If $f=6^{+}$-face: The charge does not change, and we have $c h^{*}(f)=c h(f)=$ $d(f)-6 \geq 0$.

Hence, every face now has a nonnegative charge. Now we consider $v$, a $k$-vertex in $G$. Recall we have $k \geq 3$. 
If $k=3$ : The charges of 3 -vertices do not change, so $\operatorname{ch}^{*}(v)=\operatorname{ch}(v)=$ $2(3)-6=0$.

If $k=4$ : By A1, $v$ gives one to every incident 3 -face. By Lemma $1, v$ can only be incident to at most two 3-faces. Hence, $\operatorname{ch}^{*}(v) \geq \operatorname{ch}(v)-2=2(4)-6-2=0$.

If $k=5$ : By A2, $v$ gives two to every incident 3-face. By Lemma 1, $v$ can only be incident to at most two 3 -faces. Hence, $\operatorname{ch}^{*}(v) \geq \operatorname{ch}(v)-2(2)=2(5)-6-4=0$.

If $k=6$ : By A3, $v$ gives two to each of the $\alpha$ incident 3-faces, and by A5, $v$ gives one to each of the $\beta$ pendent 3-faces. So we have $\operatorname{ch}^{*}(v)=\operatorname{ch}(v)-(2 \alpha+\beta)=$ $2(6)-6-(2 \alpha+\beta) \geq 2(6)-6-6=0$ by Lemma 1 .

If $7 \leq k \leq 10$ : Let $\alpha_{3}$ denote the number of incident faces that $v$ gives three to, $\alpha_{2}$ denote the number of incident faces $v$ gives two to, $\alpha_{1.5}$ denote the number of incident faces $v$ gives $\frac{3}{2}$ to, $\alpha_{1}$ denote the number of incident faces $v$ gives one to, and $\beta$ denote the number of pendent 3-faces of $v$. The new charge of $v$ will be negative only if we have one of the 11 cases shown in Table 2.1.

\begin{tabular}{|c|c|c|c|c|c|c|}
\hline Case & $\mathrm{k}$ & $\alpha_{3}$ & $\alpha_{2}$ & $\alpha_{1.5}$ & $\alpha_{1}$ & $\beta$ \\
\hline 1 & 7 & 2 & 0 & 0 & 0 & 3 \\
\hline 2 & 7 & 2 & 1 & 0 & 0 & 1 \\
\hline 3 & 7 & 2 & 0 & 1 & 0 & 1 \\
\hline 4 & 7 & 3 & 0 & 0 & 0 & 0 \\
\hline 5 & 7 & 3 & 0 & 0 & 0 & 1 \\
\hline 6 & 8 & 3 & 0 & 0 & 0 & 2 \\
\hline 7 & 8 & 3 & 1 & 0 & 0 & 0 \\
\hline 8 & 8 & 3 & 0 & 1 & 0 & 0 \\
\hline 9 & 8 & 4 & 0 & 0 & 0 & 0 \\
\hline 10 & 9 & 4 & 0 & 0 & 0 & 1 \\
\hline 11 & 10 & 5 & 0 & 0 & 0 & 0 \\
\hline
\end{tabular}

Table 2.1. Forbidden Cases

Cases 2 and 7 were proven not to exist by Lemmas 4 and 5 . If the $\alpha_{2}$ face is referring to either a $(3,4, k)$-star-face or a $(3,3, k)$-non-star-face, then Lemma 4 tells us these cases cannot exist. The only other time a vertex of degree 7 or 
8 gives two to a face is when $v$ is special, and the face is a $(3, j, k)$-star-face with $7 \leq j \leq 10$. However, due to the fact that in Cases 2 and 7 , our vertex is also incident to $(k-5)(3,3, k)$-star-faces and has a pendent 3 -face if $k=7, v$ is a full vertex. By Lemma 5, we cannot have a vertex that is both special and full, meaning these cases cannot exist.

Cases 5 and 9 were proven not to exist by Lemma 4 .

Cases 3 and 8 cannot exist, because the only time a 7 - or 8 -vertex gives $\frac{3}{2}$ to a face is when the face is a $(3, j, k)$-star-face with $7 \leq k \leq 8$ and $7 \leq j \leq 10$ and the vertex is neither special nor full, but the $k$-vertices in Cases 3 and 8 are incident to $(k-5)(3,3, k)$-star-faces and the vertex in Case 3 has a pendent 3 -face, making them both full vertices, a contradiction.

To show the remaining cases do not exist, we will prove that at least one of the $(3,3, k)$-star-faces is in fact a $(3,3, k)$-non-star-face, a contradiction.

We prove Case 4 does not exist first. Let $v$ be the vertex in Case 4 . Let $c$ be a $(3,0,0)$-coloring of $G \backslash\left\{v, N^{\prime}(v)\right\}$, where $N^{\prime}(v)$ denotes the set of neighbors of $v$ with degree 3. If every neighbor of $v$ has degree three, we are in Lemma 4, and hence, this configuration cannot exist in $G$. Hence, assume the degree of the vertex not incident to one of the $(3,3,7)$-star-faces has degree at least four and call it $u$. We extend this $(3,0,0)$-coloring to all $w \in N^{\prime}(v)$ such that each $w$ is properly colored. This is possible since they have at most two neighbors already colored. At most four of the neighbors of $v$ are colored by 1 . If there are exactly four neighbors colored by 1 , we have the same setup as Lemma 4 with four neighbors colored by 1 (substituting $j \geq 4$ in Case 2 , which does not change the proof).

Hence, we may assume there are three or less neighbors colored by 1 . If $c(u) \neq 1$, we may color $v$ by 1 , creating a $(3,0,0)$-coloring of $G$, since each neighbor of $v$ colored by 1 is properly colored. Thus, we can assume $c(u)=1$. If a vertex 
$x$ is colored by 2 and its neighbor on the 3-face it shares with $v$ is colored by 1 , we may recolor $x$ either by 1 (if it's outer neighbor is colored by 3 ) or by 3 (if its outer neighbor is colored by 1). Thus, we can assume the only vertices colored by 2 are incident to a $(3,3,7)$-star-face that is not incident to a vertex colored by 1 . For simplicity's sake, we will assume we only have one of these vertices. If we have more, we can color them in the same manner as follows. Let $w$ be the neighbor of $v$ colored 2 and $f$ be $w$ 's incident 3-face. Label the outer neighbor of $w$ with respect to $f$ by $z$. We are now as in Figure 2.5.

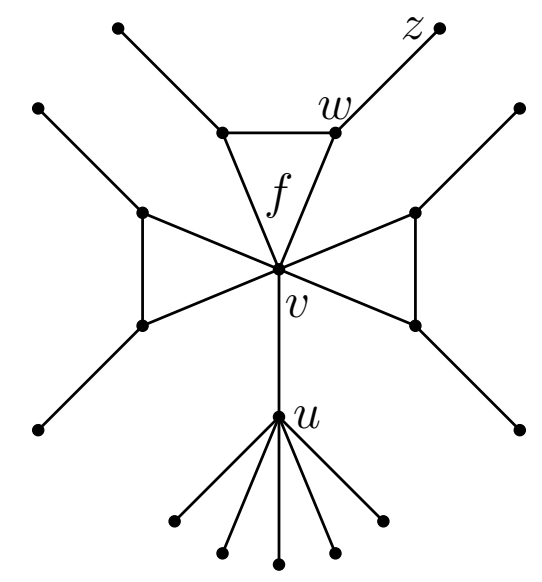

Figure 2.5. A vertex $v$ as in Case 4 of Table 2.1

If $c(z)=3$, we may recolor $w$ by 1 and then color $v$ by 2 , creating a $(3,0,0)$ coloring of $G$, a contradiction. So we must have $c(z)=1$. If $z$ is nicely colored, we may recolor $w$ by 1 and $v$ by 2 , a contradiction. Hence, $z$ must have three neighbors colored by 1 . If we can recolor $z$ by either 2 or 3 , we can then recolor $w$ by 1 and $v$ by 2, a contradiction. Hence, $z$ must have a neighbor colored by 3 and a neighbor other than $w$ colored by 2 , which implies $d(z) \geq 6$, contradicting the fact that $f$ is a $(3,3,7)$-star-face. Hence, Case 4 cannot exist in $G$.

We show the remaining Cases $(1,6,10,11)$ together. Let $v$ be a vertex as in Cases $1,6,10$, or 11 . Let $c$ be a $(3,0,0)$-coloring of $G \backslash\{v, N(v)\}$. We can extend the coloring to the neighbors of $v$ such that each neighbor is properly colored (this 
is possible since each $u \in N(v)$ has at most two colored neighbors). Let $M$ denote the set of vertices $u \in N(v)$ colored by 1 .

If $|M| \leq 3$, we can color $v$ by 1 , and therefore have a $(3,0,0)$-coloring of $G$, a contradiction.

Notice that the size of $M$ is at most five, (at most one vertex in each $(3,3, k)$ face can be colored 1 , and the remaining vertices not in a $(3,3, k)$-face can be colored 1).

First, assume $|M|=5$. If each vertex not in $M$ is colored the same color, we can color $v$ the third color, thus extending the coloring to a $(3,0,0)$-coloring of $G$, a contradiction. So the remaining $(k-5)$ neighbors of $v$ must use two colors. Note that these neighbors must be in the $(3,3, k)$-faces. Let $x$ be a neighbor of $v$ colored by 2 . Label the neighbor of $x$ in the $(3,3, k)$-face by $y$ (note that $c(y)=1$ ), and label the outer neighbor of $x$ by $w$. We can recolor $x$ by $\{1,3\} \backslash\{(c w)\}$. This can be done with any neighbor of $v$ colored 2. So after recoloring them, the neighbors of $v$ are colored either 1 or 3 , and we can then color $v$ by 2 , a contradiction. Hence, $|M| \neq 5$.

So we must have $|M|=4$. First, assume every $(3,3, k)$-star-face is incident to a vertex colored by 1 (note that this is impossible if $k=10$ ). If all $u \in N(v) \backslash M$ have the same color, we can color $v$ by the third color, a contradiction. So the remaining $(k-4)$ neighbors must use both colors 2 and 3 . There exists a unique vertex not in a $(3,3, k)$-face that is not colored 1 . Without loss of generality, assume it is colored by 3 .

Let $u$ be a vertex in a $(3,3, k)$-star-face colored 2 . We can recolor $u$ in the same manner as above (when $|M|=5$ ), so that $c(u) \in\{1,3\}$, and we can do this for all $u \in N(v)$ with $c(u)=2$. We can then color $v$ by 2 , extending this coloring to a $(3,0,0)$-coloring of $G$, a contradiction. 
Thus, there exists a unique $(3,3, k)$-star-face not incident to a vertex colored by 1 . Let the said face be $u, w, v$ with $c(u)=2$ and $c(w)=3$. Let $z$ be the outer neighbor of $u$. We can recolor any $x \in N(v) \backslash\{u\}$ with $c(x)=2$ as before so that $c(x) \in\{1,3\}$, leaving us with $u$ being the only neighbor of $v$ colored 2 .

First, assume $c(z) \neq 1$. We can then recolor $u$ by 1 and color $v$ by 2 , a contradiction. So we must have $c(z)=1$. If $z$ is nicely colored, we can recolor $u$ by 1 and color $v$ by 2 , thus extending the $(3,0,0)$-coloring to $G$. Therefore, $z$ must have three neighbors colored 1. If we can recolor $z$ by either 2 or 3 , we can recolor $u$ by 1 , and then color $v$ by 2 , a contradiction. So $z$ must have a neighbor other than $u$ colored by 2 and a neighbor colored by 3. Thus, the degree of $z$ must be at least six, making this $(3,3, k)$-star-face a $(3,3, k)$-non-star-face, a contradiction. Hence, these remaining cases cannot exist, making the charges of each $k$-vertex with $7 \leq k \leq 10$ nonnegative.

If $k \geq 11: \mathrm{By} \mathrm{A} 4, v$ gives three to each of the $\alpha$ incident 3-faces, and by A5, $v$ gives one to each of the $\beta$ pendent 3 -faces.

So we have $\operatorname{ch}^{*}(v)=\operatorname{ch}(v)-(3 \alpha+\beta)=2 k-6-\alpha-(2 \alpha+\beta) \geq 2 k-6-\alpha-k=$ $k-6-\alpha \geq k-6-\lfloor k / 2\rfloor=\lceil k / 2\rceil-6 \geq 0$.

\subsection{Conclusion}

We have now found that the charges on all the faces and vertices in $G$ are nonnegative, contradicting the fact that the sum of the charges is -12 . Hence, the graph $G$ cannot exist. Since $G$ was a minimal counterexample, no counterexample exists, and we have now proven Theorem 1 , that all graphs in $\mathcal{F}$ are $(3,0,0)$ colorable.

\section{List of References}

[1] G. Chang, F. Havet, M. Montassier, and A. Raspaud, "Steinberg's conjecture and near colorings," manuscript. 


\section{CHAPTER 3}

\section{$(1,0,0)$-Colorability}

\subsection{Introduction}

We work exclusively with finite, simple, planar graphs. We will need a few definitions before giving our main theorem. We define a cycle with two triangular chords as 2-chorded. A $k$-face is a face incident to $k$ vertices, including multiplicity if an incident vertex is a cut-vertex, which is a vertex that if removed, makes the graph disconnected. We consider $\mathcal{G}$, the family of planar graphs with no $C_{4}$ 's, no $C_{5}$ 's, no 2-chorded $C_{8}$ 's, and no 2-chorded $C_{9}$ 's. See Figure 3.1 for an example of forbidden graphs. Let a $b_{k}$-face $(k \geq 8)$ be a $k$-face that is incident with a 3 -vertex, which is itself also incident with faces of size 3 and 6 . See Figure 3.2 for an example. We now state our main theorem.

Theorem 1. Every graph in $\mathcal{G}$ with at most eleven $b_{8}$-faces is $(1,0,0)$-colorable.
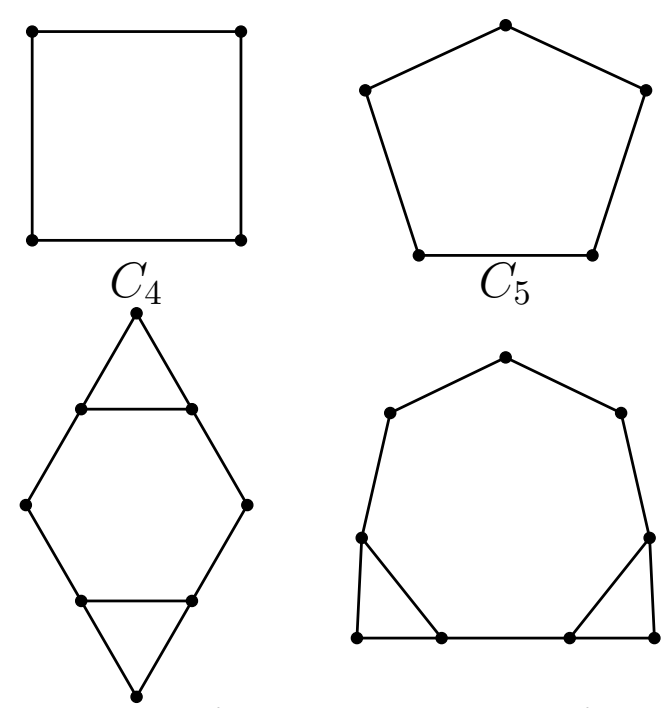

Example of a 2-chorded $C_{8}$

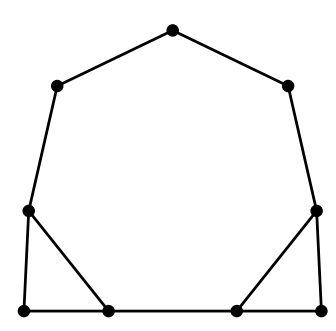

Example of a 2-chorded $C_{9}$

Figure 3.1. Forbidden graphs in $\mathcal{G}$ 
We begin with some definitions and placing charges on the faces and vertices of a minimal counterexample, $G$. In Section 3.3, we introduce some lemmas dealing with necessary configurations as well as forbidden subgraphs in this counterexample. Lastly, we discharge the charges and reach a contradiction, proving this counterexample cannot exist and thus proving Theorem 1.

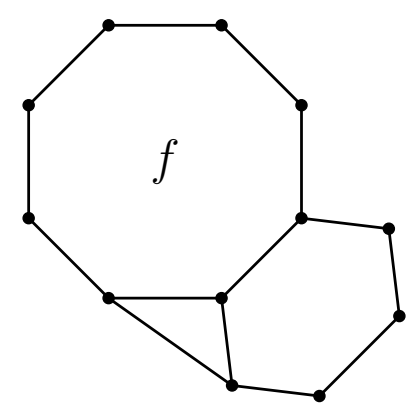

Figure 3.2. A $b_{8}$-face, $f$

\subsection{Definitions and Charges}

Let $G$ be a minimal counterexample to Theorem 1 on number of vertices, that is, $G \in \mathcal{G}$ with at most eleven $b_{8}$-faces that is not $(1,0,0)$-colorable, but $G-v$ for any $v \in V(G)$ is $(1,0,0)$-colorable. Notice first that $G$ is connected and has minimum degree at least 3 .

A $k$-face that is not bounded by a $k$-cycle is called a bad $k$-face. Note that because there are no 4 - or 5 -cycles in $G$ and the minimum degree is at least 3 , there are no 4- or 5-faces in $G$. The only bad 6-faces in $G$ are of the type shown in Figure 3.3 (where the bad face is the outer face). There are no bad 7 -faces in $G$, and the only bad 8-faces in $G$ are of the type shown in Figure 3.4 (also shown as the outer face). The only bad 9-faces in $G$ are of the types shown in Figure 3.5. From here on, the dashed lines in figures represent the possible existence of more edges.

A $\left(d_{1}, d_{2}, d_{3}\right)$-face is a 3 -face with vertices $v_{1}, v_{2}, v_{3}$ such that $d\left(v_{i}\right)=d_{i}$ for 


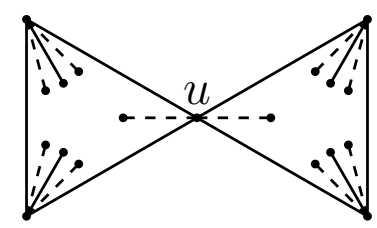

Figure 3.3. A bad 6-face

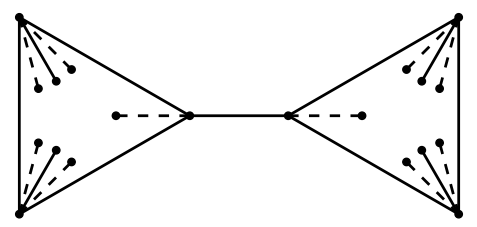

Figure 3.4. A bad 8-face

$i=1,2,3$, where $d\left(v_{i}\right)$ is the degree of $v_{i}$. Similarly, $d_{i}$ can be replaced with $d_{i}^{+}$, meaning $\mathrm{d}\left(v_{i}\right) \geq d_{i}$ or $d_{i}^{-}$, meaning $\mathrm{d}\left(v_{i}\right) \leq d_{i}$. We call a $\left(3,3,3^{+}\right)$-face where the outer neighbor of each 3-vertex is of degree 3 a poor-face.

Let $B(k)$ be the set of 3 -vertices that are incident to faces of size 3,6 , and $k$. Observe that the two 6 -faces adjacent to $v \in B(6)$ must be distinct (otherwise there are too many edges). Vertices in these $B(k)$ sets will become important in the discharging procedures in Section 3.4.

A $b_{6}$-face is defined to be a 6 -face that is incident to at least one vertex $v \in B(6) \cup B(9)$. Notice that a $b_{k}$-face, $k \geq 8$ is now also defined as a $k$-face that is incident to at least one $v \in B(k)$. It is clear that a $b_{6}$-face cannot be a bad 6 -face, as any triangle adjacent to it would create a 4-cycle. A bad 9-face of Type 1 cannot be incident to any $v \in B(9)$, as the resultant 3-face would create a 4-cycle. A bad 9-face of Type 2 can be incident to at most two $v \in B(9)$ (otherwise, there would be two 3-faces adjacent to the 6-cycle, creating a 2-chorded 8-cycle, or there would exist a 4-cycle).

Let $f$ be a $\left(3,3,5^{+}\right)$-face such that the outer neighbor of one of the 3 -vertices is degree 3 , the outer neighbor of the other 3 -vertex is at most 4 , and $f$ is adjacent to two $b_{6}$-faces and a $b_{j}$-face, with $j=6$ or 9 as in Figure 3.6. We call such a face 


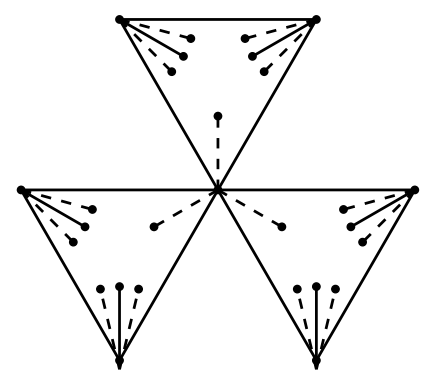

Type 1

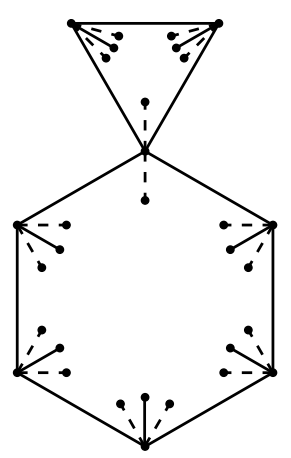

Type 2

Figure 3.5. Two bad 9-faces

a $3^{*}$-face. We call the face $g$ (shown in Figure 3.6) a special $b_{6}$-face. The outer neighbors of the 3-vertices on $f$ cannot be incident to 3-faces, as $g$ can only be adjacent to one 3 -face, namely $f$. Let $G(k)$ be the set of $k$-vertices, $k \geq 3$, that have incident and pendent faces as seen in Figure 3.7 (where $j=8$ or 9 ).

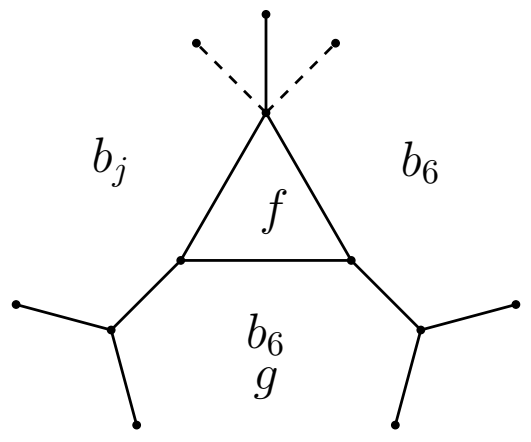

Figure 3.6. A $3^{*}$-face $f(j=6$ or 9$\left.)\right)$

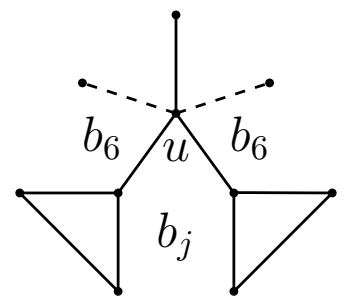

Figure 3.7. A vertex $u \in G(k)(j=8$ or 9$)$

We now assign each face and vertex of $G$ a charge, denoted by $c h$. Let $F$ denote the set of faces in $G$ and $V$ denote the set of vertices in $G$. We start with 
$\operatorname{ch}(x)=d(x)-4$ for $x \in V \cup F$, where $d(x)$ is the number of vertices incident to $x$ (counting multiplicities if $x$ is incident to a cut-vertex) for $x \in F$. By Euler's Formula, $\sum_{x \in V \cup F} \operatorname{ch}(x)=-8$.

After establishing some properties of $G$, we will redistribute the charges to the faces and vertices, while never changing the total charge. Afterwards, we will show that the new charge of each face and vertex is such that the sum cannot equal -8 . Therefore, this graph $G$ cannot exist, thus proving Theorem 1, that all graphs in $\mathcal{G}$ with at most eleven $b_{8}$ faces are $(1,0,0)$-colorable.

\subsection{Lemmas}

We now give some lemmas describing necessary and forbidden configurations in $G$.

Lemma 1. The outer neighbor of every 3 -vertex in a $\left(3,3,4^{-}\right)$-face in $G$ has degree at least 4.

Proof. Let $f=[u, v, w]$ be a $\left(3,3,4^{-}\right)$-face, that is, $d(u)=d(v)=3$ and $3 \leq$ $d(w) \leq 4$. Without loss of generality, we can assume $d(w)=4$. Label the outer neighbor of $u$ by $u^{\prime}$ and the outer neighbor of $v$ by $v^{\prime}$. For a contradiction, assume $d\left(u^{\prime}\right)=3$. Let $c$ be a $(1,0,0)$-coloring of $G \backslash\left\{u, v, w, u^{\prime}\right\}$. Properly color $v, w$, and $u^{\prime}$. This is possible as each vertex has at most two neighbors colored when we color it. If $v, w$, and $u^{\prime}$ use only two colors, we can properly color $u$, a contradiction. Hence, we can assume each of these vertices uses a different color. If $c(v)=1$ or $c\left(u^{\prime}\right)=1$, we may color $u$ by 1 , creating a $(1,0,0)$-coloring of $G$, a contradiction. Thus, $c(w)=1$. Without loss of generality, assume $c\left(u^{\prime}\right)=2$ and $c(v)=3$. If the remaining neighbors of $w$ do not use the color 1 , we can color $u$ by 1 . Hence, one of the neighbors of $w$ is colored 1 . If the last neighbor of $w$ is colored by 3 , we can recolor $w$ by 2 and properly color $u$ by 1 . Hence, the remaining neighbor of $w$ must be colored by 2 . We can then recolor $w$ by 3 , recolor $v$ by $\{1,2,\} \backslash\left\{c\left(v^{\prime}\right)\right\}$, and color 
$u$ by 1 . We can do the same for $v$ if $d\left(v^{\prime}\right)=3$, and for $w$ if we assume $d(w)=3$. Since this contradicts the fact that $G$ is not $(1,0,0)$-colorable, this configuration cannot exist in $G$, and the outer neighbors of the 3 -vertices in a $\left(3,3,4^{-}\right)$-face in $G$ must have degree at least four.

Lemma 2. Let $v$ be a 5-vertex in $G$ incident to two $(3,3,5)$-faces. The outer neighbors of both of the 3-vertices on one of the $(3,3,5)$-faces must have degree at least four.

Proof. For a contradiction, assume at least one 3-vertex on each $(3,3,5)$-face has an outer neighbor of degree three. Let the vertices be labeled as in Figure 3.8, where $d\left(x^{\prime}\right)=d\left(y^{\prime}\right)=3$.

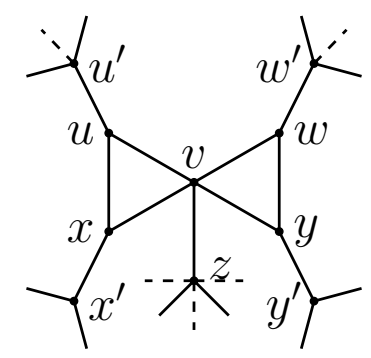

Figure 3.8. Impossible configuration, as in Lemma 2

Let $c$ be a $(1,0,0)$-coloring of $G \backslash\{u, v, w, x, y\}$. Properly color $u, w, x, y$. This is possible as each vertex has at most two neighbors colored when we color it. At most three neighbors of $v$ are colored by $1(z$ and one neighbor in each 3-face). If exactly three neighbors are colored by 1, we may assume the remaining two neighbors are colored differently, otherwise we could then properly color $v$. Suppose, without loss of generality, that vertex $u$ is colored by 2 . We can recolor $u$ by $\{1,3\} \backslash\left\{c\left(u^{\prime}\right)\right\}$ and then color $v$ by 2 . This is a $(1,0,0)$-coloring of $G$, a contradiction.

We next assume two neighbors of $v$ are colored by 1 . We break this into two cases, depending on the color of $z$. 
Case 1: $c(z)=1$.

We can assume the vertices on one of the 3-faces are colored using the colors 1 and 2, while the other face uses the colors 2 and 3 . Without loss of generality, assume the face with vertices $w$ and $y$ use the colors 2 and 3 . If $c(y)=3$, we recolor $y$ by 1. The only possible problem with this would be if $c\left(y^{\prime}\right)=1$ and $y^{\prime}$ already has a neighbor colored by 1 . In this case, we recolor $y^{\prime}$ by the color not used on its neighbors. Since $d\left(y^{\prime}\right)=3$, we know such a color exists. If $c(w)=3$, we recolor $w$ by $\{1,2\} \backslash\left\{c\left(w^{\prime}\right)\right\}$. If the color of $w$ is now 2 , we recolor $y$ by 1 . As above, the only problem would occur if $c\left(y^{\prime}\right)=1$ and it has a neighbor other than $y$ colored 1 , in which case we color $y^{\prime}$ with the color not used on its neighbors. In either situation, we may now color $v$ by 3 , a contradiction, proving this case cannot exist.

Case 2: $c(z) \neq 1$.

Without loss of generality, assume $c(z)=2$. One vertex in each incident $(3,3,5)$ face is colored by 1 . Assume $c(w)=3$. We recolor $w$ by $\{1,2\} \backslash\left\{c\left(w^{\prime}\right)\right\}$. We do the same thing to $u$ or $x$ if either of them are colored by 3 . We can then color $v$ by 3 , a contradiction.

Now, we may assume at most one neighbor of $v$ is colored by 1 . If $c(z) \neq 1$, we may color $v$ by 1 , a contradiction. Hence, we must have $c(z)=1$. Notice this implies that the vertices on each 3 -face are colored by 2 and 3 . We again break this into two cases.

Case 3: Two vertices in the $(3,3,5)$-faces with outer neighbors of degree 3 are colored the same color.

Without loss of generality, assume $c(x)=c(y)=2$. We recolor $x$ and $y$ by 1 . If $c\left(x^{\prime}\right)=1$ and already has a neighbor colored by 1 , we recolor $x^{\prime}$ by the color not used on its neighbors, and we do the same for $y^{\prime}$ if necessary. We can then color $v$ by 2 , a contradiction. Hence, this case cannot exist. 
Case 4: $d\left(u^{\prime}\right) \geq 4, d\left(w^{\prime}\right) \geq 4$, and $c(u)=c(y)$.

Without loss of generality, assume $c(u)=3$. We recolor $u$ by $\{1,2\} \backslash\left\{c\left(u^{\prime}\right)\right\}$. If $u$ is now colored by 2 , we can recolor $x$ by 1 , recoloring $x^{\prime}$ if needed as above. We then recolor $y$ by 1 , again recoloring $y^{\prime}$ if needed as above. We can now color $v$ by 3, reaching a contradiction. Hence this case cannot exist.

We have now shown that both outer neighbors on at least one of the $(3,3,5)$ faces incident to a 5 -vertex must have degree at least four.

Lemma 3. A 6- or 7-face in $G$ cannot be adjacent to more than one 3-face.

Proof. It creates either a 2-chorded $C_{8}$ or a 2-chorded $C_{9}$.

Lemma 4. No 3-faces in $G$ may be adjacent.

Proof. It creates a 4-cycle.

Lemma 5. A 6-face $f$ in $G$ cannot be incident to six 3-vertices.

Proof. We break this into two cases.

Case 1: $f$ is a bad 6-face.

We know there exists a $4^{+}$-vertex in $f$ (vertex $u$ in Figure 3.3).

Case 2: $f$ is bounded by a 6 -cycle.

For a contradiction, suppose such a 6 -face $f$ exists. Let the vertices on $f$ be labeled $v_{1}, v_{2}, \ldots, v_{6}$ in a counterclockwise order. Label the vertex adjacent to $v_{i}$ but not on $f$ by $v_{i}^{\prime}$ for $1 \leq i \leq 6$. Choose a $(1,0,0)$-coloring of $G \backslash\left\{v_{1}, v_{2}, \ldots, v_{6}\right\}$. If each $v_{i}^{\prime}$ is colored the same color, we may alternate the colors of each $v_{i}$ using the two remaining colors. Hence, we may assume that $v_{1}^{\prime}$ and $v_{2}^{\prime}$ use two different colors. Color $v_{2}$ by the color used on $v_{1}^{\prime}$, and then proceed to color the vertices in a counterclockwise manner, starting with $v_{3}$. There will always be at least one unused color to choose from to color $v_{i}, 1 \leq i \leq 6$. 


\subsection{Discharging}

We discharge the charges in the following manner:

Charges from a vertex:

V1: A 4-vertex gives $\frac{1}{2}$ to every incident 3 -face.

V2: A 5 -vertex gives $\frac{7}{6}$ to every incident $3^{*}$-face, 1 to every remaining incident $(3,3,5)$-poor-face, $\frac{2}{3}$ to every remaining incident $(3,3,5)$-non-poor-face, and $\frac{1}{2}$ to every other incident 3-face.

$V 3$ : A $6^{+}$-vertex gives $\frac{7}{6}$ to every incident $3^{*}$-face and 1 to every other 3 -face.

$V 4$ : A $4^{+}$-vertex gives $\frac{1}{3}$ to every pendent 3 -face.

Charges from a face:

$F 1$ : A $b_{6}$-face gives $\frac{1}{2}$ to every incident $v \in B(6), \frac{7}{18}$ to every incident $v \in B(9), \frac{1}{3}$ to every other 3 -vertex, and $\frac{1}{6}$ to every incident $4^{+}$-vertex.

F2: A 7 -face gives $\frac{2}{3}$ to every incident 3 -vertex that is also incident to a 3 -face and $\frac{1}{3}$ to every other incident vertex.

F3: A $b_{8}$-face gives $\frac{2}{3}$ to every incident $v \in B(8), \frac{4}{9}$ to every incident $v \in G(k)$, and $\frac{1}{2}$ to every other incident vertex.

F4: A $b_{9}$-face gives $\frac{11}{18}$ to every incident $v \in B(9), \frac{4}{9}$ to every incident $v \in G(k)$, and $\frac{1}{2}$ to every other incident vertex.

F5: A $b_{k}$-face, $10 \leq k \leq 11$, gives $\frac{2}{3}$ to every incident $v \in B(k)$ and $\frac{1}{2}$ to every other incident vertex.

F6: Every remaining $6^{+}$-face $f$ gives $\frac{c h(f)}{d(f)}$ to every incident vertex.

F7: A $3^{*}$-face gives $\frac{1}{6}$ to the adjacent special $b_{6}$-face.

If $f$ is a bad $k$-face, then it is incident to some cut-vertex $v$, where $v$ has multiplicity $n$ on $f$. Suppose $f$ gives a charge equal to $m$ to $v$ by one of the rules above. Then in total, $v$ receives $n m$ from $f$.

This does not come into play when $f$ is a 3-face or when $v \in B(k)$ for any $k$. 
As stated before, a bad 6-face cannot be a $b_{6}$-face, and any vertex $v \in B(k)$ for $k \geq 8$ is incident to three distinct face sizes.

\subsection{New Charges}

Let the new charge be denoted by $c h^{*}$. First, we prove the new charge on each $7^{-}$-face is non-negative.

Let $\boldsymbol{f}$ be a 3 -face. The initial charge of $f$ is $\operatorname{ch}(f)=d(f)-4=-1$. Notice that if $f$ is not a $3^{*}$-face, it does not give charge to anything.

$f=\left(3,3,4^{-}\right)$: The outer neighbor of each 3 -vertex has degree at least four by Lemma 1 , so they each give $f \frac{1}{3}$ by $V 4$. If the third vertex is a 4 -vertex, it gives $\frac{1}{2}$ by $V 1$, otherwise it is a 3 -vertex, and its outer neighbor gives $\frac{1}{3}$ to $f$ by $V 4$. So, $c h^{*}(f) \geq-1+3\left(\frac{1}{3}\right)=0$.

$f=(3,3,5):$

Case 1: $f$ is a $3^{*}$-face.

The 5 -vertex gives $\frac{7}{6}$ to $f$ by $V 2$ and $f$ gives $\frac{1}{6}$ to the special $b_{6}$-face by $F 7$, giving $c h^{*}(f) \geq-1+\frac{7}{6}-\frac{1}{6}=0$.

Case 2: $f$ is a $(3,3,5)$-poor-face that is not a $3^{*}$-face.

The 5 -vertex gives 1 to $f$ by $V 2$, so $c^{*}(f)=-1+1=0$.

Case $3: f$ is a $(3,3,5)$-non-poor-face that is not a $3^{*}$-face.

At least one of the 3 -vertices on $f$ has an outer neighbor of degree at least four, and so that vertex gives $\frac{1}{3}$ to $f$ by $V 4$. The 5 -vertex gives $\frac{2}{3}$ to $f$ by $V 2$, leaving $c h^{*}(f) \geq-1+\frac{1}{3}+\frac{2}{3}=0$.

$f=\left(3,3,6^{+}\right)$:

Case $1: f$ is a $3^{*}$-face.

The $6^{+}$-vertex gives $\frac{7}{6}$ to $f$ by $V 3$ and $f$ gives $\frac{1}{6}$ to the special $b_{6}$-face by $F 7$, so $c h^{*}(f) \geq-1+\frac{7}{6}-\frac{1}{6}=0$.

Case $2: f$ is not a $3^{*}$-face. 
The $6^{+}$-vertex gives 1 to $f$ by $V 3$, so $c h^{*}(f) \geq-1+1=0$.

$f=\left(3^{+}, 4^{+}, 4^{+}\right)$: Each $4^{+}$-vertex gives at least $\frac{1}{2}$ to $f$ by $V 1-V 3$, so $\operatorname{ch}^{*}(f) \geq$ $-1+2\left(\frac{1}{2}\right)=0$.

\section{There are no 4- or 5-faces.}

Let $\boldsymbol{f}$ be a 6 -face. The initial charge of $f$ is $\operatorname{ch}(f)=d(f)-4=2$.

Case 1: $f$ is not a $b_{6}$-face.

We have $c h^{*}(f)=2-6\left(\frac{2}{6}\right)=0$ by $F 6$.

Case 2: $f$ is a $b_{6}$-face.

We know that it can be adjacent to at most one 3-face (otherwise it creates a 2-chorded 8-cycle), so it can be incident to at most two vertices $v \in B(6) \cup B(9)$.

We know there exists a $4^{+}$-vertex on $f$ by Lemma 5 . If $f$ is incident to only one $v \in B(6) \cup B(9)$, then $f$ gives at most $\frac{1}{2}$ to it, $\frac{1}{6}$ to the $4^{+}$-vertex, and at most $\frac{1}{3}$ to the remaining four vertices by $F 1$, which leaves $c h^{*}(f) \geq 2-\frac{1}{2}-\frac{1}{6}-4\left(\frac{1}{3}\right)=0$.

Suppose $f$ is incident to two $v \in B(9)$. Then $f$ gives $\frac{7}{18}$ to each of them, $\frac{1}{6}$ to the $4^{+}$-vertex, and at most $\frac{1}{3}$ to the remaining three vertices, leaving $c h^{*}(f) \geq$ $2-2\left(\frac{7}{18}\right)-\frac{1}{6}-2\left(\frac{1}{3}\right)=\frac{7}{18}>0$.

Next, suppose $f$ is incident to one $v \in B(6)$ and one $v \in B(6) \cup B(9)$. We know these two vertices must be incident to the same 3 -face, call it $g$, adjacent to $f$, since $f$ can only be adjacent to one 3 -face. If the third vertex on $g$ has degree 3 or 4 , then by Lemma 1 , we know that the outer neighbor of each of the 3 -vertices has degree at least four, which means $f$ gives $\frac{1}{2}$ to the vertex $v \in B(6)$, at most $\frac{1}{2}$ to the other vertex $u \in B(6) \cup B(9), \frac{1}{6}$ to each $4^{+}$-vertex, and at most $\frac{1}{3}$ to the remaining two vertices. This gives $c h^{*}(f) \geq 2-2\left(\frac{1}{2}\right)-2\left(\frac{1}{6}\right)-2\left(\frac{1}{3}\right)=0$.

So we can assume the third vertex on $g$ has degree at least five. If $g$ is a $3^{*}$-face, $g$ gives $\frac{1}{6}$ to $f$ by $F 7$, and we get $c h^{*}(f) \geq 2-2\left(\frac{1}{2}\right)-\frac{1}{6}-3\left(\frac{1}{3}\right)+\frac{1}{6}=0$. Otherwise, both outer neighbors of the 3-vertices on $g$ have degree at least four, 
giving us $c h^{*}(f) \geq 2-2\left(\frac{1}{2}\right)-2\left(\frac{1}{6}\right)-2\left(\frac{1}{3}\right)=0$.

Let $\boldsymbol{f}$ be a 7 -face. The initial charge of $f$ is $\operatorname{ch}(f)=d(f)-4=3$.

The face $f$ gives $\frac{2}{3}$ to any incident 3 -vertex that is also incident to a 3 -face, of which, $f$ can be incident to at most two. The face also gives $\frac{1}{3}$ to every remaining vertex. Hence, $c h^{*}(f) \geq 3-2\left(\frac{2}{3}\right)-5\left(\frac{1}{3}\right)=0$.

We hold off on looking at faces of size 8 for now. We have a few more lemmas before we look at $9^{+}$-faces.

Lemma 6. Let $f$ be a $b_{k}$-face, $k \geq 8$, in $G$. Any two consecutive vertices in $B(k)$ on $f$ must form a 3-face.

Proof. Suppose they do not. Then they must have the form of Figure 3.9.

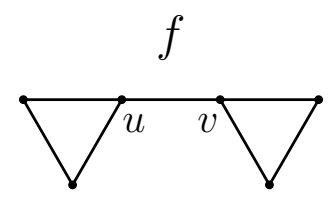

Figure 3.9. Impossible configuration, as in Lemma 6, where $u, v \in B(k)$

However, this gives us a 6-face adjacent to two 3-faces, contradicting Lemma 3.

Corollary 1. A $b_{k}$-face, $k \geq 8$, cannot have three consecutive vertices in $B(k)$.

Proof. There must exist two consecutive vertices in $B(k)$ not on the same 3 -face.

Corollary 2. A $b_{k}$-face, $k \geq 8$, cannot be incident to more than $\left\lfloor\frac{2 k}{3}\right\rfloor$ vertices in $B(k)$.

Proof. There exist three consecutive vertices in $B(k)$.

We now prove the charges on $9^{+}$-faces are nonnegative. 
Let $\boldsymbol{f}$ be a 9-face. The initial charge of $f$ is $\operatorname{ch}(f)=d(f)-4=5$.

Case 1: $f$ is not a $b_{9}$-face.

We have $c h^{*}(f)=5-9\left(\frac{5}{9}\right)=0$ by $F 6$.

Case 2: $f$ is a $b_{9}$-face.

Suppose $f$ is incident to $n$ vertices in $B(9)$. By Corollary 2, we know $n \leq 6$.

Suppose $n \leq 4$ :

The face $f$ gives $\frac{11}{18}$ to the $n$ vertices in $B(9)$ and at most $\frac{1}{2}$ to the remaining vertices by $F 4$, leaving $c h^{*}(f) \geq 5-n\left(\frac{11}{18}\right)-(9-n)\left(\frac{1}{2}\right)=\frac{9-2 n}{18}>0$.

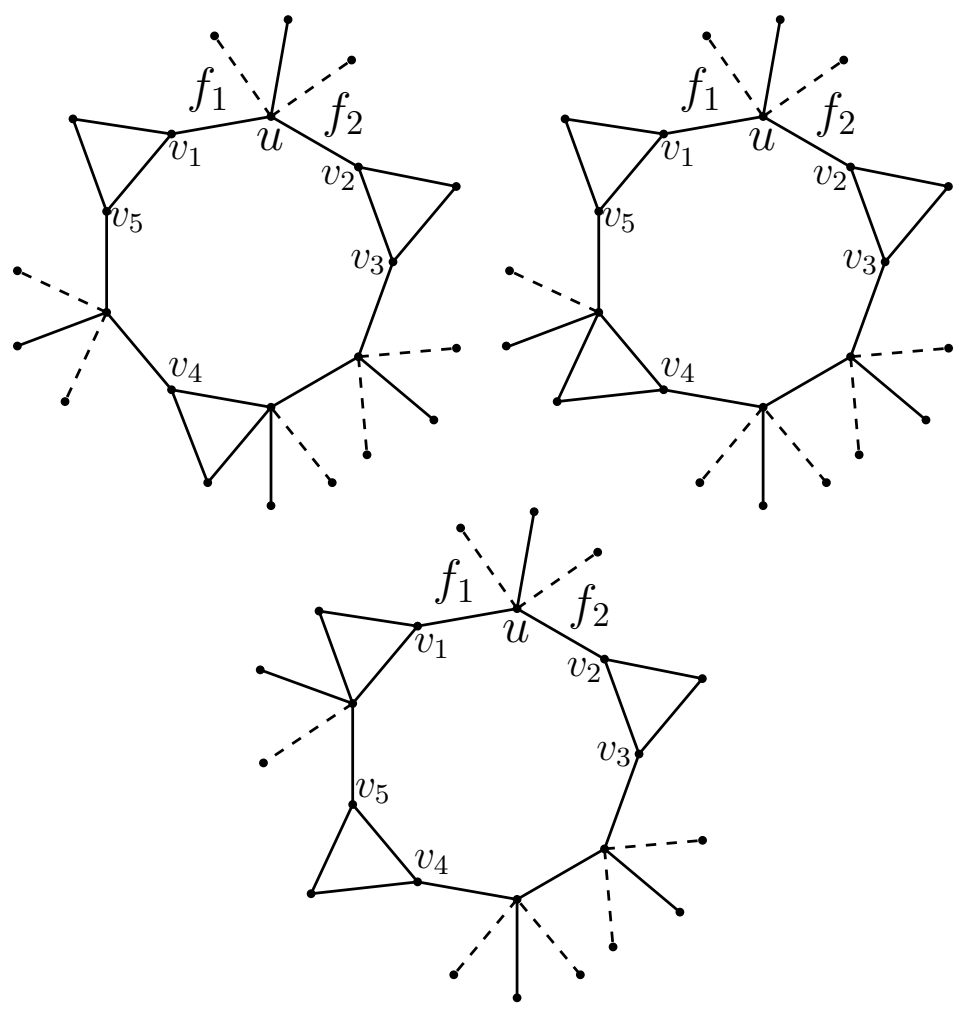

Figure 3.10. All possible 9-faces with five vertices in $B(9)$ (labeled $v_{1}, v_{2}, \ldots, v_{5}$ )

Suppose $n=5$ :

Notice that $f$ must be bounded by a 9-cycle. There are three possible configurations, see Figure 3.10. In all three cases, $f$ is incident to at least one vertex $u \in G(k)$, since $f_{1}$ and $f_{2}$ must both be $b_{6}$-faces. This gives 
$c h^{*}(f) \geq 5-5\left(\frac{11}{18}\right)-3\left(\frac{1}{2}\right)-\frac{4}{9}=0$ by $F 4$.

Suppose $n=6$.

Again, notice that $f$ must be bounded by a 9-cycle. There is only one possible configuration for this, see Figure 3.11. Notice all three vertices not in $B(9)$ must be in $G(k)$ for some values of $k$, since we must have $d\left(f_{1}\right)=d\left(f_{2}\right)=\ldots=d\left(f_{6}\right)=6$. This gives $c h^{*}(f)=5-6\left(\frac{11}{18}\right)-3\left(\frac{4}{9}\right)=0$ by $F 4$.

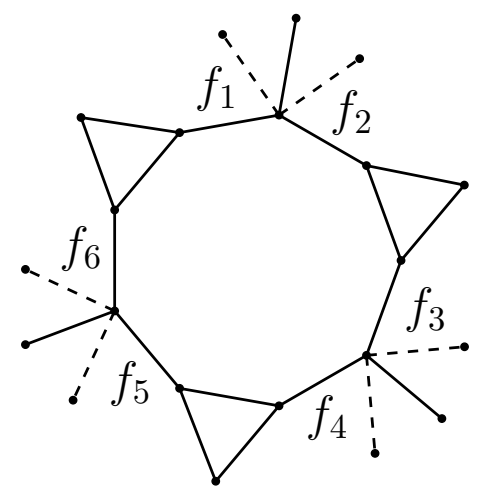

Figure 3.11. A 9-face with six vertices in $B(9)$

Let $f$ be a $\boldsymbol{k}$-face with $10 \leq \boldsymbol{k} \leq \mathbf{1 1}$. The initial charge of $f$ is $\operatorname{ch}(f)=$ $d(f)-4=k-4$.

Case 1: $f$ is not a $b_{k}$-face.

We have $c h^{*}(f)=c h(f)-d(f) \frac{c h(f)}{d(f)}=0$ by $F 6$.

Case 2: $f$ is a $b_{k}$-face.

Let $f$ be incident to $n$ vertices in $B(k)$. We know by Corollary $2, n \leq\left\lfloor\frac{2 k}{3}\right\rfloor$. If $k=10, n \leq 6$, and if $k=11, n \leq 7$, giving us $n \leq k-4$ in either case. This means $c h^{*}(f) \geq k-4-n\left(\frac{2}{3}\right)-(k-n)\left(\frac{1}{2}\right)=\frac{3 k-n-24}{6} \geq \frac{2 k+4-24}{6}=\frac{2 k-20}{6} \geq 0$ by $F 5$.

\section{Let $f$ be a $12^{+}$-face.}

We have $c h^{*}(f)=\operatorname{ch}(f)-d(f) \frac{\operatorname{ch}(f)}{d(f)}=0$ by $F 6$.

Hence, all the faces, except possibly some 8-faces, have nonnegative charge. We now look at the charges on each vertex. Since a face gives some amount of 
charge to a vertex every time the vertex is counted on the face, we may assume from here on that the faces incident to a vertex are distinct, that is, a $k$-vertex is incident to $k$ faces.

Let $\boldsymbol{v}$ be a 3-vertex. The initial charge of $v$ is $\operatorname{ch}(v)=d(v)-4=-1$.

Case 1: $v \in B(6)$.

Each incident $b_{6}$-face gives $v \frac{1}{2}$ by $F 1$. This gives $c h^{*}(v)=-1+2\left(\frac{1}{2}\right)=0$.

Case 2: $v \in B(8)$.

The 6 -face gives $v \frac{1}{3}$ by $F 1$ or $F 6$, and the $b_{8}$-face gives $\frac{2}{3}$ by $F 3$, which leaves $c h^{*}(v)=-1+\frac{1}{3}+\frac{2}{3}=0$.

Case 3: $v \in B(9)$.

The $b_{6}$-face gives $v \frac{7}{18}$ by $F 1$ and the $b_{9}$-face gives $\frac{11}{18}$ by $F 4$, so $c h^{*}(v)=-1+\frac{7}{18}+$ $\frac{11}{18}=0$.

Case 4: $v \in B(k)$ with $10 \leq k \leq 11$.

The 6 -face gives $\frac{1}{3}$ by $F 1$ or $F 6$, and the $b_{k}$-face gives $\frac{2}{3}$ by $F 5$, which leaves $c h^{*}(v)=-1+\frac{1}{3}+\frac{2}{3}=0$.

Case 5: $v \notin B(k)$ for any $k \leq 11$ but is incident to a 3 -face.

Let the two remaining incident faces be labeled $f_{1}$ and $f_{2}$. They are either of degree 6 and 7, of degree 6 and at least 12, or both of degree at least 7 . In the first two instances, the 6-face gives $v \frac{1}{3}$ and the other face gives $v$ at least $\frac{2}{3}$, leaving $c h^{*}(v) \geq-1+\frac{1}{3}+\frac{2}{3}=0$. In the last instance, both faces give at least $\frac{1}{2}$, leaving $c h^{*}(v) \geq-1+2\left(\frac{1}{2}\right)=0$.

Case 6: $v$ is not incident to a 3 -face.

Each face incident to $v$ is of degree at least six, which means each face gives at least $\frac{1}{3}$ by $F 1-F 6$. We get $c h^{*}(v) \geq-1+3\left(\frac{1}{3}\right)=0$.

Let $\boldsymbol{v}$ be a 4-vertex. The initial charge of $v$ is $\operatorname{ch}(v)=d(v)-4=0$.

Case 1: $v$ is not incident to a 3 -face. 
If $v$ has two or less pendent 3 -faces, then all the faces incident to $v$ are $6^{+}$-faces, which give at least $\frac{1}{6}$ to $f$ by $F 1-F 6$, giving us $c h^{*}(v) \geq-2\left(\frac{1}{3}\right)+4\left(\frac{1}{6}\right)=0$.

If $v$ has 3 or 4 pendent 3 -faces, then $v$ must be incident to at least two $8^{+}$-faces, each of which gives at least $\frac{1}{2}$ to $v$, and $v$ is incident to at most two 6- or 7-faces, each of which gives at least $\frac{1}{6}$. This gives $c h^{*}(v) \geq-4\left(\frac{1}{3}\right)+2\left(\frac{1}{2}\right)+2\left(\frac{1}{6}\right)=0$.

Case 2: $v$ is incident to one 3 -face.

If $v$ has no pendent 3 -face, then the remaining faces are $6^{+}$-faces which give at least $\frac{1}{6}$ to $v$, giving us $c h^{*}(v) \geq-\frac{1}{2}+3\left(\frac{1}{6}\right)=0$.

If $v$ has one pendent 3-face, then at least one of the faces incident to $v$ is an $8^{+}$-face, leaving $c h^{*}(v) \geq-\frac{1}{2}-\frac{1}{3}+\frac{1}{2}+2\left(\frac{1}{6}\right)=0$.

If $v$ has two pendent 3-faces, then the other three faces $v$ is incident to must be $8^{+}$-faces, and $c h^{*}(v) \geq-\frac{1}{2}-2\left(\frac{1}{3}\right)+3\left(\frac{1}{2}\right)=\frac{1}{3}>0$.

Case 3: $v$ is incident to two 3-faces.

Each of the other two incident faces must be $8^{+}$-faces. Thus, $\operatorname{ch}^{*}(v) \geq-2\left(\frac{1}{2}\right)+$ $2\left(\frac{1}{2}\right)=0$.

Let $\boldsymbol{v}$ be a 5-vertex. The initial charge of $v$ is $\operatorname{ch}(v)=d(v)-4=1$.

Case 1: $v$ is not incident to a 3 -face.

We have at most five pendent 3 -faces and five incident $6^{+}$-faces, giving us $c h^{*}(v) \geq$ $1-5\left(\frac{1}{3}\right)+5\left(\frac{1}{6}\right)=\frac{1}{6}>0$.

Case 2: $v$ is incident to one 3-face.

The incident 3 -face receives at most $\frac{7}{6}$ from $v$. If $v$ has one or less pendent 3 -faces, then the other four incident faces are $6^{+}$-faces and $c h^{*}(v) \geq 1-\frac{7}{6}-\frac{1}{3}+4\left(\frac{1}{6}\right)=\frac{1}{6}>0$.

If $v$ has two pendent 3 -faces, then $v$ must be incident to at least two $8^{+}$-faces, and $c h^{*}(v) \geq 1-\frac{7}{6}+2\left(\frac{1}{2}\right)+2\left(\frac{1}{6}\right)=\frac{1}{2}>0$.

If $v$ has three pendent 3 -faces, then $v$ must be incident to four $8^{+}$-faces, giving $c h^{*}(v) \geq 1-\frac{7}{6}-3\left(\frac{1}{3}\right)+4\left(\frac{1}{2}\right)=\frac{5}{6}>0$. 
Case 3: $v$ is incident to two 3-faces.

Suppose $v$ does not have a pendent 3-face. By Lemma 2, at most one of the incident 3 -faces may be a $3^{*}$-face. Also by Lemma 2 , both 3 -faces cannot be poor. So, $v$ gives at most $\frac{7}{6}$ to at most one of the 3 -faces, and $v$ gives at most $\frac{2}{3}$ to the remaining incident 3 -face. One of the remaining incident faces must be an $8^{+}$-face, giving $c h^{*}(v) \geq 1-\frac{7}{6}-\frac{2}{3}+\frac{1}{2}+2\left(\frac{1}{6}\right)=0$.

If $v$ has a pendent 3 -face, then all three of the remaining faces must be $8^{+}$ faces, and so neither of the incident 3 -faces can be $3^{*}$-faces. Thus, $v$ gives at most 1 to each face by $V 2$, giving us $c h^{*}(v) \geq 1-2(1)-\frac{1}{3}+3\left(\frac{1}{2}\right)=\frac{1}{6}>0$.

Let $\boldsymbol{v}$ be a $\boldsymbol{k}$-vertex with $\boldsymbol{k} \geq \mathbf{6}$. Suppose $v$ is incident to $\alpha 3$-faces. Note that $\alpha \leq\left\lfloor\frac{k}{2}\right\rfloor$. The initial charge of $v$ is $\operatorname{ch}(v)=d(v)-4=k-4$.

Case 1: $v \in G(k)$ incident to $n\left(b_{6}, b_{8}, b_{6}\right)$ or $\left(b_{6}, b_{9}, b_{6}\right)$ face combinations.

Notice that $\alpha \leq\left\lfloor\frac{k-3 n-1}{2}\right\rfloor$, since each of the $b_{6}$-faces next to the $b_{8^{-}}$or $b_{9^{-}}$ face cannot be adjacent to any 3 -faces on $v$ (since they are already adjacent to a pendent 3 -face of $v$ ).

Each incident 3 -face receives at most $\frac{7}{6}$ from $v$ by $V 3$, each pendent 3-face (of which there are at most $(k-2 \alpha))$ receives $\frac{1}{3}$ from $v$ by $V 4$, each of the $n b_{8^{-}}$or $b_{9}$-faces of the $\left(b_{6}, b_{8}, b_{6}\right)$ or $\left(b_{6}, b_{9}, b_{6}\right)$ variety give $\frac{4}{9}$ to $v$ by $F 3$ or $F 4$, and each of the remaining $(k-\alpha-n)$ faces are $6^{+}$-faces, giving at least $\frac{1}{6}$ to $v$ by $F 1-F 6$. This gives:

$$
\begin{aligned}
c h^{*}(v) & \geq k-4-\alpha\left(\frac{7}{6}\right)-(k-2 \alpha)\left(\frac{1}{3}\right)+n\left(\frac{4}{9}\right)+(k-\alpha-n)\left(\frac{1}{6}\right) \\
& =\frac{5}{6} k-\frac{2}{3} \alpha+\frac{5}{18} n-4 \\
& =\frac{1}{18}(15 k-12 \alpha+5 n-72) \\
& \geq \frac{1}{18}\left(15 k-12\left(\frac{k-3 n-1}{2}\right)+5 n-72\right) \\
& =\frac{1}{18}(9 k+23 n-66)
\end{aligned}
$$




$$
\begin{aligned}
& \geq \frac{1}{18}(9 k+23-66) \\
& =\frac{1}{18}(9 k-43) \\
& >0
\end{aligned}
$$

Case 2: $v \notin G(k)$ and $k=6$.

Case 2A: $\alpha=0$.

The vertex $v$ can have at most six pendent 3 -faces, and all incident faces give at least $\frac{1}{6}$ to $v$ by $F 1-F 6$, leaving $c h^{*}(v) \geq 2-6\left(\frac{1}{3}\right)+6\left(\frac{1}{6}\right)=1>0$.

Case 2B: $\alpha=1$.

The vertex $v$ gives at most $\frac{7}{6}$ to the 3-face by $V 3, v$ has at most four pendent 3-faces, and the five remaining faces each give at least $\frac{1}{6}$ to $v$. Thus, $\operatorname{ch}^{*}(v) \geq$ $2-\frac{7}{6}-4\left(\frac{1}{3}\right)+5\left(\frac{1}{6}\right)=\frac{1}{3}>0$.

Case 2C: $\alpha=2$.

If $v$ has no pendent 3 -faces, then $v$ is incident to four $6^{+}$-faces, giving $\operatorname{ch}^{*}(v) \geq$ $2-2\left(\frac{7}{6}\right)+4\left(\frac{1}{6}\right)=\frac{1}{3}>0$.

If $v$ has one or two pendent 3-faces, then $v$ must be incident to at least two $8^{+}$-faces, giving $c h^{*}(v) \geq 2-2\left(\frac{7}{6}\right)-2\left(\frac{1}{3}\right)+2\left(\frac{1}{2}\right)+2\left(\frac{1}{6}\right)=\frac{1}{3}>0$.

Case 2D: $\alpha=3$.

All remaining faces must be $8^{+}$-faces, and we get $c h^{*}(v) \geq 2-3\left(\frac{7}{6}\right)+3\left(\frac{1}{2}\right)=0$.

Case 3: $v \notin G(k)$ and $k \geq 7$.

Case 3A: $\alpha \leq\left\lfloor\frac{k}{3}\right\rfloor$.

Each incident 3 -face receives at most $\frac{7}{6}$ from $v$ by $V 3$, each pendent 3-face (of which there are at most $(k-2 \alpha))$ receive $\frac{1}{3}$ from $v$ by $V 4$, and each of the remaining $(k-\alpha)$ faces give at least $\frac{1}{6}$ to $v$ by $F 1-F 6$, making $c h^{*}(v) \geq k-4-\alpha\left(\frac{7}{6}\right)-(k-$ $2 \alpha)\left(\frac{1}{3}\right)+(k-\alpha)\left(\frac{1}{6}\right)=\frac{5}{6} k-\frac{2}{3} \alpha-4 \geq \frac{5}{6} k-\frac{2}{3}\left(\frac{k}{3}\right)-4=\frac{11}{18} k-4>0$.

Case 3B: $\alpha>\left\lfloor\frac{k}{3}\right\rfloor$.

At least $(3 \alpha-k)$ incident faces of $v$ must be $8^{+}$-faces. This is because every time 
four consecutive neighbors of $v$ form two 3-faces, the face between them must be an $8^{+}$-face. The vertex $v$ may have up to $(k-2 \alpha)$ pendent 3 -faces, and the remaining $(2 k-4 \alpha)$ faces are $6^{+}$-faces. Thus, $c h^{*}(v) \geq k-4-\alpha\left(\frac{7}{6}\right)-(k-2 \alpha)\left(\frac{1}{3}\right)+(3 \alpha-$ $k)\left(\frac{1}{2}\right)+(2 k-4 \alpha)\left(\frac{1}{6}\right)=\frac{1}{2} k+\frac{1}{3} \alpha-4 \geq \frac{1}{2} k+\frac{1}{3}\left(\frac{k}{3}\right)-4=\frac{11}{18} k-4>0$.

We have now shown that the new charge on each vertex in $G$ is non-negative. We look at the new charge on each 8-face next.

Let $\boldsymbol{f}$ be an 8-face. The initial charge of $f$ is $\operatorname{ch}(f)=d(f)-4=4$.

Case 1: $f$ is not a $b_{8}$-face.

We have $c h^{*}(f)=4-8\left(\frac{4}{8}\right)=0$ by $F 6$.

Case 2: $f$ is a $b_{8}$-face.

Suppose $f$ is incident to $n$ vertices in $B(8)$. By Corollary 2, we know $n \leq 5$.

Suppose $n \leq 4$ :

The face $f$ gives $\frac{2}{3}$ to the $n$ vertices in $B(8)$ and at most $\frac{1}{2}$ to the remaining vertices.

Thus, $c h^{*}(f) \geq 4-n\left(\frac{2}{3}\right)-(8-n)\left(\frac{1}{2}\right)=-\frac{n}{6} \geq-\frac{2}{3}$.

Suppose $n=5$ :

There is only one possible configuration, see Figure 3.12. At least two of the remaining incident vertices must be in $G(k)$ for some $k\left(w_{1}\right.$ and $w_{2}$ in Figure 3.12) since $d\left(f_{1}\right)=d\left(f_{2}\right)=d\left(f_{3}\right)=d\left(f_{4}\right)=6$, giving us $c h^{*}(f) \geq 4-5\left(\frac{2}{3}\right)-2\left(\frac{4}{9}\right)-\frac{1}{2}=$ $-\frac{13}{18}$

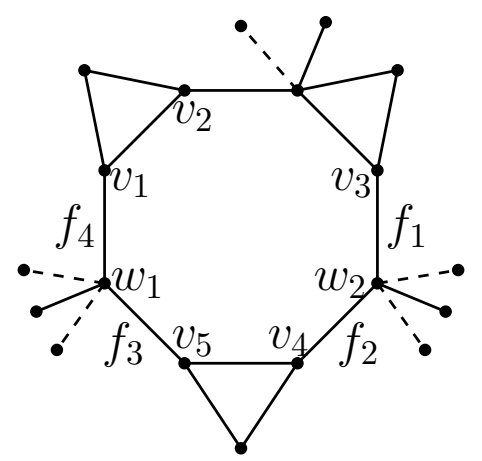

Figure 3.12. An 8-face with five vertices in $B(8)$ (labeled $v_{1}, v_{2}, \ldots, v_{5}$ ) 
We will now show a contradiction to the fact that the sum of the charges must equal -8 . Recall that at most eleven $b_{8}$ faces exist in $G$. Then we have:

$$
\begin{aligned}
-8 & =\sum_{v \in V} c h^{*}(v)+\sum_{f \in F} c h^{*}(f) \geq \sum_{f \in F} c h^{*}(f) \\
& =\sum_{\substack{f \in F \\
d(f) \neq 8}} c h^{*}(f)+\sum_{\substack{f \in F \\
d(f)=8}} c h^{*}(f) \\
& \geq \sum_{\substack{f \in F \\
f \text { is a } b_{8} \text {-face }}} c h^{*}(f) \\
& \geq 11\left(-\frac{13}{18}\right) \\
& =-\frac{143}{18}
\end{aligned}
$$

This is a contradiction. This completes the proof of Theorem 1.

\subsection{Conclusion}

While Steinberg's Conjecture is still open, much progress has been made over the past few years. $\mathrm{Bu}, \mathrm{Xu}$, and Wang [1] have compiled the progress on $(1,0,0)$ colorability of planar graphs without certain short cycles. There are papers that

claim for every triple $(4, j, k)$, planar graphs without cycles of length $4, j$, or $k$ are $(1,0,0)$-colorable whenever $4<j<k \leq 9$. Mondal [2, 3] claimed to have proven that all planar graphs without cycles of length 4, 5, and 8 are 3-colorable and further that all planar graphs without 5- and 8-cycles and without adjacent 3cycles are also 3-colorable. Chapter 4, however, will give reasons why these proofs of Mondal's are invalid.

\section{List of References}

[1] Y. Bu, J. Xu, and Y. Wang, "(1, 0, 0)-colorability of planar graphs without prescribed short cycles," Journal of Combinatorial Optimization, pp. 1-20, 2013. 
[2] S. Mondal, "Planar graphs without 4-, 5- and 8-cycles are 3-colorable." Discussiones Mathematicae: Graph Theory, vol. 31, pp. 775-789, 2011.

[3] S. Mondal, "Planar graphs without 5- and 8-cycles and adjacent triangles are 3-colorable," Journal of Combinatorial Mathematics and Combinatorial Computing, vol. 81, pp. 81-95, 2012. 


\section{CHAPTER 4}

\section{Future Work}

\subsection{Planar Graphs with no 5- or 8-Cycles or Adjacent 3-Cycles}

In 2012, Mondal [1] claimed to have proven that all planar graphs without cycles of length 5 or 8 and without adjacent triangles are 3-colorable. Several flaws exist in the proof. We have managed to correct most of them.

In the paper, he attempts to prove that any proper 3-coloring of a given certain type of cycle of a planar graph without 5- and 8-cycles and without adjacent triangles can be extended to the whole graph. He uses a minimality argument similar to Chapters 2 and 3. He lets $G$ be a minimal (on number of vertices and edges) counterexample to his theorem.

He gives charges to the vertices and faces and then list a discharging method, just as in Chapters 2 and 3. However, the charges he assigns to the vertices $(d(v)-6)$ and the faces $\left(2 d(f)-6\right.$ for $f \in F(G) \backslash f_{0}$ and $d\left(f_{0}\right)+\frac{9}{2}$ where $f_{0}$ is the outer face of $G$ ) in the second paragraph of Section 3 [1] do not coincide with the charges he actually uses $\left(d(x)-4\right.$ for $x \in V(G) \cup F(G) \backslash f_{0}$ and $\left.d\left(f_{0}\right)+4\right)$. In addition, when he attempted to show the new weights are nonnegative for all $x \in V(G) \cup F(G)$ and the new weight of the outer face is positive, he ignores some of the discharging rules. Most significantly, we show here that there exist multiple faces which, according to the discharging methods he used, have a negative final charge.

We now give some counterexamples to his claims that each face has a nonnegative final charge. The following are the discharging procedures used throughout his paper:

R0. Each 3 -face $f=x y z$ receives $\frac{1}{3}$ from each adjacent face.

R1. Every 3 -vertex $v \notin C$ receives $\frac{1}{3}$ from each incident face, unless $v$ is incident 
with one 3 -face, in which case $v$ receives $\frac{1}{2}$ from each of the two $>3$-faces.

R2. Every 2-vertex receives $\frac{5}{3}$ from the external face, and $\frac{1}{3}$ from the other adjacent (i.e. internal) face.

R3. The external face $f_{0}$ gives 1 to each incident vertex of degree at least 3 .

R4. Let $v_{1}, v_{2}, v_{3}$ be consecutive vertices of external face $f_{0}$ with $d\left(v_{2}\right) \geq 4$. Then $v_{2}$ gives 1 to each incident face not incident with edges $v_{1} v_{2}$ and $v_{2} v_{3}$. Furthermore, if the internal face receiving 1 is a 3 -face $\left(v_{2} x y\right)$ where $x$ and $y$ do not belong to $f_{0}$, then it passes the 1 to the neighboring internal face.

R5. Each $9^{+}$-face $f \neq f_{0}$ gives $\frac{d(f)-8}{2}$ to $f_{0}$.

By the definition given in [1], a tetrad is the configuration shown in Figure 4.1 , where $d\left(v_{1}\right)=d\left(v_{2}\right)=d\left(v_{3}\right)=d\left(v_{4}\right)=3$ and each of the vertices $v_{1}, v_{2}, v_{3}, v_{4}$ are interior (i.e., not on the external face). In the paper, he uses the fact that a $k$-face $f$ cannot be incident with a tetrad. However, he is ignoring the cases when a pseudo-tetrad appears, where a pseudo-tetrad is a tetrad such that any of the vertices $v_{1}, v_{2}, v_{3}, v_{4}$ may be on the external face. This would increase the charge that $f$ gives away, making it possible that the final charge is negative. See Figure 4.2 for an example, where the dashed lines represent edges on $C$.

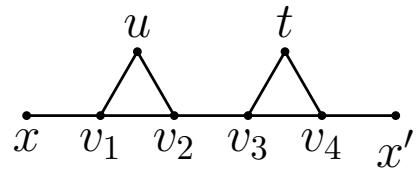

Figure 4.1. A tetrad

See Figures 4.3 and 4.4 for more examples of internal faces with negative final charge that are not incident with a tetrad of any kind.

We would also like to point out that Mondal [2] has a paper claiming to prove that all planar graphs without cycles of length 4,5 , and 8 are 3 -colorable. The proof is very similar to this one and also has several flaws in it. A major flaw is 


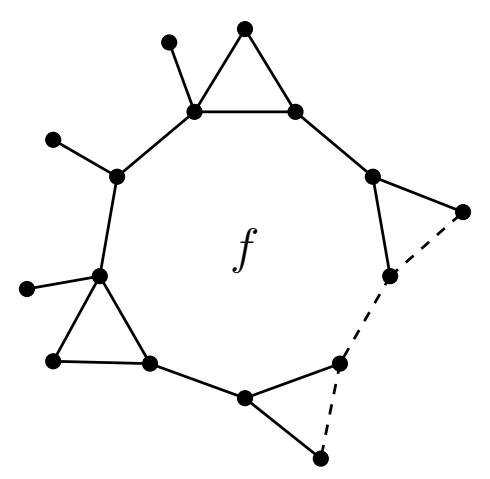

Figure 4.2. An internal face incident with a pseudo-tetrad where $w^{\prime}(f)=-\frac{1}{6}$

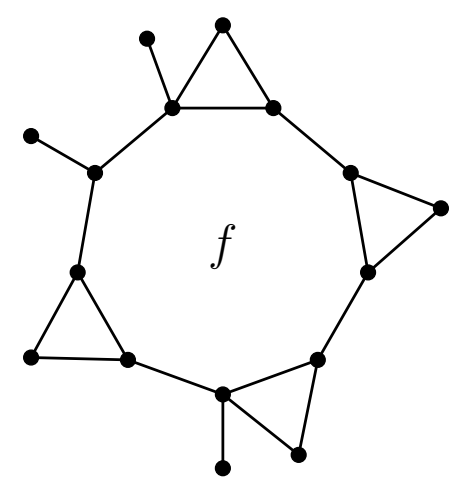

Figure 4.3. An internal 9-face $f$ with $w^{\prime}(f)=-\frac{1}{6}$ not incident with a tetrad

in the proof of the claim that there are no 6-faces other than possibly the external face (like Claim 12 in [1]). He starts off by saying that if there does exist a 6 -face $f$, it cannot be adjacent to a triangle. The proof goes as follows:

"If $f$ has an adjacent 3-cycle, we remove the common edge between $f$ and the 3-cycle. The resulting graph is smaller than $G$, and does not have any 4-, 5- or 8-cycle. So we assume that $G$ does not have any adjacent 3-cycle."

He ignores the fact that he must show he can extend the coloring of $G-e$ to $G$, which would be impossible if the two end-vertices of the edge are colored the same. He also only states, and does not prove, that collapsing the 6 -face by identifying two certain vertices cannot make the external face bad, which is a term he uses in both [2] and [1]. 


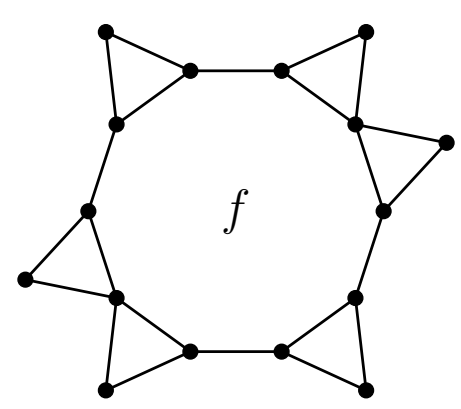

Figure 4.4. An internal 10-face $f$ with $w^{\prime}(f)=-1$ not incident with a tetrad

\subsection{Steinberg's Conjecture}

We would like to classify graphs which have no 4 - and 5-cycles and minimum degree 3 , and therefore gain an understanding of the structure of such a graph were there to exist a minimal counterexample to Steinberg's Conjecture. We already know that these graphs must be 2-connected and have minimum degree at least three. By a discharging procedure, we proved that we can narrow down the list of potential counterexamples by looking at the structural properties of faces of sizes 6 through 9. We know that certain kinds of cycles must exist in such a graph. In that way, we narrow down the possibilities for minimum counter examples. For example, we know that we cannot have an even cycle incident to all vertices of degree three. The proof of this is the same as the proof of Lemma 5 in Chapter 3. Of course, it is our ultimate goal to one day either prove or disprove Steinberg's Conjecture as a whole.

\section{List of References}

[1] S. Mondal, "Planar graphs without 5- and 8-cycles and adjacent triangles are 3-colorable," Journal of Combinatorial Mathematics and Combinatorial Computing, vol. 81, pp. 81-95, 2012.

[2] S. Mondal, "Planar graphs without 4-, 5- and 8-cycles are 3-colorable." Discussiones Mathematicae: Graph Theory, vol. 31, pp. 775-789, 2011. 


\section{BIBLIOGRAPHY}

Abbott, H. and Zhou, B., "On small faces in 4-critical graphs," Ars Combinatoria, pp. 203-207, 1991.

Appel, K. and Haken, W., "Every planar map is four colorable. Part I. Discharging," Illinois Journal of Mathematics, pp. 429-490, 1977.

Appel, K. and Haken, W., "Every planar map is four colorable. Part II. Reducibility," Illinois Journal of Mathematics, pp. 491-567, 1977.

Borodin, O., "Structural properties of plane graphs without adjacent triangles and an application to 3-colorings," Journal of Graph Theory, vol. 21, pp. 183-186, 1996.

Borodin, O., Glebov, A., Raspaud, A., and Salavatipour, M., "Planar graphs without cycles of length from 4 to 7 are 3-colorable," Journal of Combinatorial Theory, pp. 303-311, 2005.

$\mathrm{Bu}, \mathrm{Y}$. and $\mathrm{Fu}, \mathrm{C}$., " $(1,1,0)$-coloring of planar graphs without cycles of length 4 and 6," Discrete Mathematics, vol. 313, pp. 2737 - 2741, 2013.

$\mathrm{Bu}, \mathrm{Y} ., \mathrm{Xu}, \mathrm{J}$. , and Wang, Y., "(1, 0, 0)-colorability of planar graphs without prescribed short cycles," Journal of Combinatorial Optimization, pp. 1-20, 2013.

Chang, G., Havet, F., Montassier, M., and Raspaud, A., "Steinberg's conjecture and near colorings," manuscript.

Cowen, L., Cowen, R., and Woodall, D., "Defective colorings of graphs in surfaces: partitions into subgraphs of bounded valency," Journal of Graph Theory, pp. 187-195, 1986.

Eaton, N. and Hull, T., "Defective list colorings of planar graphs," Bulletin of the Institute of Combinatorics and its Applications, pp. 79-87, 1999.

Grötzsch, H., "Ein dreifarbensatz für dreikreisfreie netze auf der kugel,," Math.Naturwiss. Reihe 8, pp. 109-120, 1959, in German.

Grünbaum, B., "Grötzsch's theorem on 3-colorings," Michigan Mathematics Journal, pp. 303-310, 1963.

Heawood, P., "Map colour theorem," Quarterly Journal of Pure and Applied Mathematics, pp. 332-338, 1890. 
Hill, O., Smith, D., Wang, Y., Xu, L., and Yu, G., "Planar graphs without cycles of length 4 or 5 are (3, 0, 0)-colorable," Discrete Mathematics, vol. 313, pp. 2312-2317, 2013.

Hill, O. and Yu, G. Arxiv. "A relaxation of Steinberg's conjecture." [Online]. Available: arxiv.org/abs/1208.3395

Lih, K., Song, Z., Wang, W., and Zhang, K., "A note on list improper coloring planar graphs," Applied Mathematics Letters, pp. 269-273, 2001.

Maritz, P. and Mouton, S., "Francis Guthrie: A colourful life," The Mathematical Intelligencer, vol. 34, pp. 67-75, 2012.

Mondal, S., "Planar graphs without 4-, 5- and 8-cycles are 3-colorable." Discussiones Mathematicae: Graph Theory, vol. 31, pp. 775-789, 2011.

Mondal, S., "Planar graphs without 5- and 8-cycles and adjacent triangles are 3colorable," Journal of Combinatorial Mathematics and Combinatorial Computing, vol. 81, pp. 81-95, 2012.

Proskurowski, A. and Syslo, M., "Efficient vertex-and edge-coloring of outerplanar graphs," Society for Industrial and Applied Mathematics Journal on Algebraic Discrete Methods, vol. 7, no. 1, pp. 131-136, 1986.

Salavatipour, M., "The three color problem for planar graphs," Department of Computer Science, University of Toronto, Toronto, Ontario, Canada, Tech. Rep., July 2002.

Sanders, D. and Zhao, Y., "A note on the three color problem," Graphs and Combinatorics, pp. 91-94, 1995.

Steinberg, R., "The state of the three color problem, quo vadis, graph theory?" Annals of Discrete Mathematics, pp. 211-248, 1993.

Škrekovski, R., "List improper coloring of planar graphs," Combinatorics Probability and Computing, pp. 293-299, 1999.

Wang, Y., Jin, L., and Kang, Y., "Planar graphs without cycles of length from 4 to 6 are (1, 0, 0)-colorable," Scientia Sinica Mathematica, vol. 43, pp. 1145-1164, 2013, in Chinese.

Wang, Y. and Xu, J., "Planar graphs with cycles of length neither 4 nor 6 are (2,0,0)-colorable," Information Processing Letters, vol. 113, pp. 659-663, 2013.

Wang, Y. and Xu, J., "Improper colorability of planar graphs without prescribed short cycles," Discrete Mathematics, vol. 322, pp. 5-14, 2014.

Wang, Y. and Yang, Y., “(1,0,0)-colorability of planar graphs without cycles of length 4, 5, or 9," 2012, submitted. 
West, D., Introduction to Graph Theory, 2nd ed. Prentice Hall, 2001, ch. 5, p. 192.

Xu, B., "On $(3,1)^{*}$-coloring of plane graphs," Society for Industrial and Applied Mathematics Journal of Discrete Mathematics, vol. 1, pp. 205-220, 2008/09.

Xu, L., Miao, Z., and Wang, Y., "Every planar graph with cycles of length neither 4 nor 5 is $(1,1,0)$-colorable," Journal of Combinatorial Optimization, DOI 10.10071s10878-012-9586-4. 